\title{
FRACTIONAL INTEGRAL INEQUALITIES INVOLVING CONVEXITY
}

\author{
GEORGE A. ANASTASSIOU
}

\author{
Dedicated to Professor Mustafa Kulenović on the occasion of his 60th birthday
}

\begin{abstract}
Here we present general integral inequalitites involving convex and increasing functions applied to products of functions. As specific applications we derive a wide range of fractional inequalities of Hardy type. These involve the left and right: Erdélyi-Kober fractional integrals, mixed Riemann-Liouville fractional multiple integrals. Next we produce multivariate Poincaré type fractional inequalitites involving left fractional radial derivatives of Canavati type, Riemann-Liouville and Caputo types. The exposed inequalities are of $L_{p}$ type, $p \geq 1$, and exponential type.
\end{abstract}

\section{INTRODUCTION}

We start with some facts about fractional derivatives needed in the sequel, for more details see, for instance [1], [10].

Let $a<b, a, b \in \mathbb{R}$. By $C^{N}([a, b])$, we denote the space of all functions on $[a, b]$ which have continuous derivatives up to order $N$, and $A C([a, b])$ is the space of all absolutely continuous functions on $[a, b]$. By $A C^{N}([a, b])$, we denote the space of all functions $g$ with $g^{(N-1)} \in A C([a, b])$. For any $\alpha \in \mathbb{R}$, we denote by $[\alpha]$ the integral part of $\alpha$ (the integer $k$ satisfying $k \leq \alpha<k+1)$, and $\lceil\alpha\rceil$ is the ceiling of $\alpha(\min \{n \in \mathbb{N}, n \geq \alpha\})$. By $L_{1}(a, b)$, we denote the space of all functions integrable on the interval $(a, b)$, and by $L_{\infty}(a, b)$ the set of all functions measurable and essentially bounded on $(a, b)$. Clearly, $L_{\infty}(a, b) \subset L_{1}(a, b)$.

We start with the definition of the Riemann-Liouville fractional integrals, see [13]. Let $[a, b],(-\infty<a<b<\infty)$ be a finite interval on the real axis $\mathbb{R}$. The Riemann-Liouville fractional integrals $I_{a+}^{\alpha} f$ and $I_{b-}^{\alpha} f$ of order $\alpha>0$

2000 Mathematics Subject Classification. 26A33, 26D10, 26D15.

Key words and phrases. Fractional integral, fractional radial derivative, Hardy fractional inequality, Poincaré fractional inequality, Erdélyi-Kober fractional integrals. 
are defined by

$$
\begin{aligned}
& \left(I_{a+}^{\alpha} f\right)(x)=\frac{1}{\Gamma(\alpha)} \int_{a}^{x} f(t)(x-t)^{\alpha-1} d t, \quad(x>a), \\
& \left(I_{b-}^{\alpha} f\right)(x)=\frac{1}{\Gamma(\alpha)} \int_{x}^{b} f(t)(t-x)^{\alpha-1} d t, \quad(x<b),
\end{aligned}
$$

respectively. Here $\Gamma(\alpha)$ is the Gamma function. These integrals are called the left-sided and the right-sided fractional integrals. We mention some properties of the operators $I_{a+}^{\alpha} f$ and $I_{b-}^{\alpha} f$ of order $\alpha>0$, see also [16]. The first result yields that the fractional integral operators $I_{a+}^{\alpha} f$ and $I_{b-}^{\alpha} f$ are bounded in $L_{p}(a, b), 1 \leq p \leq \infty$, that is

$$
\left\|I_{a+}^{\alpha} f\right\|_{p} \leq K\|f\|_{p}, \quad\left\|I_{b-}^{\alpha} f\right\|_{p} \leq K\|f\|_{p},
$$

where

$$
K=\frac{(b-a)^{\alpha}}{\alpha \Gamma(\alpha)} .
$$

Inequality (3), that is the result involving the left-sided fractional integral, was proved by H. G. Hardy in one of his first papers, see [11]. He did not write down the constant, but the calculation of the constant was hidden inside his proof.

Next we follow [12].

Let $\left(\Omega_{1}, \Sigma_{1}, \mu_{1}\right)$ and $\left(\Omega_{2}, \Sigma_{2}, \mu_{2}\right)$ be measure spaces with positive $\sigma$-finite measures, and let $k: \Omega_{1} \times \Omega_{2} \rightarrow \mathbb{R}$ be a nonnegative measurable function, $k(x, \cdot)$ measurable on $\Omega_{2}$ and

$$
K(x)=\int_{\Omega_{2}} k(x, y) d \mu_{2}(y), \quad x \in \Omega_{1} .
$$

We suppose that $K(x)>0$ a.e. on $\Omega_{1}$, and by a weight function (shortly: a weight), we mean a nonnegative measurable function on the actual set. Let the measurable functions $g: \Omega_{1} \rightarrow \mathbb{R}$ with the representation

$$
g(x)=\int_{\Omega_{2}} k(x, y) f(y) d \mu_{2}(y),
$$

where $f: \Omega_{2} \rightarrow \mathbb{R}$ is a measurable function.

Theorem 1. ([12]) Let $u$ be a weight function on $\Omega_{1}, k$ a nonnegative measurable function on $\Omega_{1} \times \Omega_{2}$, and $K$ be defined on $\Omega_{1}$ by (5). Assume that the function $x \mapsto u(x) \frac{k(x, y)}{K(x)}$ is integrable on $\Omega_{1}$ for each fixed $y \in \Omega_{2}$. Define $\nu$ on $\Omega_{2}$ by

$$
\nu(y):=\int_{\Omega_{1}} u(x) \frac{k(x, y)}{K(x)} d \mu_{1}(x)<\infty .
$$


If $\Phi:[0, \infty) \rightarrow \mathbb{R}$ is convex and increasing function, then the inequality

$$
\int_{\Omega_{1}} u(x) \Phi\left(\left|\frac{g(x)}{K(x)}\right|\right) d \mu_{1}(x) \leq \int_{\Omega_{2}} \nu(y) \Phi(|f(y)|) d \mu_{2}(y)
$$

holds for all measurable functions $f: \Omega_{2} \rightarrow \mathbb{R}$ such that:

(i) $f, \Phi(|f|)$ are both $k(x, y) d \mu_{2}(y)$-integrable, $\mu_{1}$-a.e. in $x \in \Omega_{1}$,

(ii) $\nu \Phi(|f|)$ is $\mu_{2}$-integrable,

and for all corresponding functions g given by (6).

Important assumptions (i) and (ii) are missing from Theorem 2.1. of [12].

In this article we use and generalize Theorem 1 for products of several functions and we give wide applications to Fractional Calculus.

\section{MAIN RESUlts}

Let $\left(\Omega_{1}, \Sigma_{1}, \mu_{1}\right)$ and $\left(\Omega_{2}, \Sigma_{2}, \mu_{2}\right)$ be measure spaces with positive $\sigma$-finite measures, and let $k_{i}: \Omega_{1} \times \Omega_{2} \rightarrow \mathbb{R}$ be nonnegative measurable functions, $k_{i}(x, \cdot)$ measurable on $\Omega_{2}$, and

$$
K_{i}(x)=\int_{\Omega_{2}} k_{i}(x, y) d \mu_{2}(y), \quad \text { for any } x \in \Omega_{1},
$$

$i=1, \ldots, m$. We assume that $K_{i}(x)>0$ a.e. on $\Omega_{1}$, and the weight functions are nonnegative measurable functions on the related set.

We consider measurable functions $g_{i}: \Omega_{1} \rightarrow \mathbb{R}$ with the representation

$$
g_{i}(x)=\int_{\Omega_{2}} k_{i}(x, y) f_{i}(y) d \mu_{2}(y),
$$

where $f_{i}: \Omega_{2} \rightarrow \mathbb{R}$ are measurable functions, $i=1, \ldots, m$.

Here $u$ stands for a weight function on $\Omega_{1}$.

The first introductory result is proved for $m=2$.

Theorem 2. Assume that the functions $(i=1,2) x \mapsto\left(u(x) \frac{k_{i}(x, y)}{K_{i}(x)}\right)$ are integrable on $\Omega_{1}$, for each fixed $y \in \Omega_{2}$. Define $u_{i}$ on $\Omega_{2}$ by

$$
u_{i}(y):=\int_{\Omega_{1}} u(x) \frac{k_{i}(x, y)}{K_{i}(x)} d \mu_{1}(x)<\infty .
$$

Let $p, q>1: \frac{1}{p}+\frac{1}{q}=1$. Let the functions $\Phi_{1}, \Phi_{2}: \mathbb{R}_{+} \rightarrow \mathbb{R}_{+}$, be convex and increasing. Then

$$
\begin{gathered}
\int_{\Omega_{1}} u(x) \Phi_{1}\left(\left|\frac{g_{1}(x)}{K_{1}(x)}\right|\right) \Phi_{2}\left(\left|\frac{g_{2}(x)}{K_{2}(x)}\right|\right) d \mu_{1}(x) \leq \\
\left(\int_{\Omega_{2}} u_{1}(y) \Phi_{1}\left(\left|f_{1}(y)\right|\right)^{p} d \mu_{2}(y)\right)^{\frac{1}{p}}\left(\int_{\Omega_{2}} u_{2}(y) \Phi_{2}\left(\left|f_{2}(y)\right|\right)^{q} d \mu_{2}(y)\right)^{\frac{1}{q}},
\end{gathered}
$$


for all measurable functions $f_{i}: \Omega_{2} \rightarrow \mathbb{R}(i=1,2)$ such that

(i) $f_{1}, \Phi_{1}\left(\left|f_{1}\right|\right)^{p}$ are both $k_{1}(x, y) d \mu_{2}(y)$-integrable, $\mu_{1}$-a.e. in $x \in \Omega_{1}$,

(ii) $f_{2}, \Phi_{2}\left(\left|f_{2}\right|\right)^{q}$ are both $k_{2}(x, y) d \mu_{2}(y)$-integrable, $\mu_{1}$-a.e. in $x \in \Omega_{1}$,

(iii) $u_{1} \Phi_{1}\left(\left|f_{1}\right|\right)^{p}, u_{2} \Phi_{2}\left(\left|f_{2}\right|\right)^{q}$, are both $\mu_{2}$-integrable,

and for all corresponding functions $g_{i}(i=1,2)$ given by $(10)$.

Proof. Notice that $\Phi_{1}, \Phi_{2}$ are continuous functions. Here we use Hölder's inequality. We have

$$
\begin{gathered}
\int_{\Omega_{1}} u(x) \Phi_{1}\left(\left|\frac{g_{1}(x)}{K_{1}(x)}\right|\right) \Phi_{2}\left(\left|\frac{g_{2}(x)}{K_{2}(x)}\right|\right) d \mu_{1}(x) \\
=\int_{\Omega_{1}} u(x)^{\frac{1}{p}} \Phi_{1}\left(\left|\frac{g_{1}(x)}{K_{1}(x)}\right|\right) u(x)^{\frac{1}{q}} \Phi_{2}\left(\left|\frac{g_{2}(x)}{K_{2}(x)}\right|\right) d \mu_{1}(x) \\
\leq\left(\int_{\Omega_{1}} u(x) \Phi_{1}\left(\left|\frac{g_{1}(x)}{K_{1}(x)}\right|\right)^{p} d \mu_{1}(x)\right)^{\frac{1}{p}} \\
\cdot\left(\int_{\Omega_{1}} u(x) \Phi_{2}\left(\left|\frac{g_{2}(x)}{K_{2}(x)}\right|\right)^{q} d \mu_{1}(x)\right)^{\frac{1}{q}}
\end{gathered}
$$

(notice here that $\Phi_{1}^{p}, \Phi_{2}^{q}$ are convex, increasing and continuous nonnegative functions, and by Theorem 1 we get)

$$
\leq\left(\int_{\Omega_{2}} u_{1}(y) \Phi_{1}\left(\left|f_{1}(y)\right|\right)^{p} d \mu_{2}(y)\right)^{\frac{1}{p}}\left(\int_{\Omega_{2}} u_{2}(y) \Phi_{2}\left(\left|f_{2}(y)\right|\right)^{q} d \mu_{2}(y)\right)^{\frac{1}{q}} .
$$

The general result follows

Theorem 3. Assume that the functions $(i=1,2, \ldots, m \in \mathbb{N}) x \mapsto(u(x)$ $\left.\cdot \frac{k_{i}(x, y)}{K_{i}(x)}\right)$ are integrable on $\Omega_{1}$, for each fixed $y \in \Omega_{2}$. Define $u_{i}$ on $\Omega_{2}$ by

$$
u_{i}(y):=\int_{\Omega_{1}} u(x) \frac{k_{i}(x, y)}{K_{i}(x)} d \mu_{1}(x)<\infty .
$$

Let $p_{i}>1: \sum_{i=1}^{m} \frac{1}{p_{i}}=1$. Let the functions $\Phi_{i}: \mathbb{R}_{+} \rightarrow \mathbb{R}_{+}, i=1, \ldots, m$, be convex and increasing. Then

$$
\begin{gathered}
\int_{\Omega_{1}} u(x) \prod_{i=1}^{m} \Phi_{i}\left(\left|\frac{g_{i}(x)}{K_{i}(x)}\right|\right) d \mu_{1}(x) \leq \\
\prod_{i=1}^{m}\left(\int_{\Omega_{2}} u_{i}(y) \Phi_{i}\left(\left|f_{i}(y)\right|\right)^{p_{i}} d \mu_{2}(y)\right)^{\frac{1}{p_{i}}},
\end{gathered}
$$

for all measurable functions $f_{i}: \Omega_{2} \rightarrow \mathbb{R}(i=1, \ldots, m)$ such that 
(i) $f_{i}, \Phi_{i}\left(\left|f_{i}\right|\right)^{p_{i}}$ are both $k_{i}(x, y) d \mu_{2}(y)$-integrable, $\mu_{1}$-a.e. in $x \in \Omega_{1}$, $i=1, \ldots, m$

(ii) $u_{i} \Phi_{i}\left(\left|f_{i}\right|\right)^{p_{i}}$ is $\mu_{2}$-integrable, $i=1, \ldots, m$, and for all corresponding functions $g_{i}(i=1, \ldots, m)$ given by $(10)$.

Proof. Notice that $\Phi_{i}, i=1, \ldots, m$, are continuous functions. Here we use the generalized Hölder's inequality. We have

$$
\begin{aligned}
& \int_{\Omega_{1}} u(x) \prod_{i=1}^{m} \Phi_{i}\left(\left|\frac{g_{i}(x)}{K_{i}(x)}\right|\right) d \mu_{1}(x) \\
= & \int_{\Omega_{1}} \prod_{i=1}^{m}\left(u(x)^{\frac{1}{p_{i}}} \Phi_{i}\left(\left|\frac{g_{i}(x)}{K_{i}(x)}\right|\right)\right) d \mu_{1}(x) \\
\leq & \prod_{i=1}^{m}\left(\int_{\Omega_{1}} u(x) \Phi_{i}\left(\left|\frac{g_{i}(x)}{K_{i}(x)}\right|\right)^{p_{i}} d \mu_{1}(x)\right)^{\frac{1}{p_{i}}}
\end{aligned}
$$

(notice here that $\Phi_{i}^{p_{i}}, i=1, \ldots, m$, are convex, increasing and continuous, nonnegative functions, and by Theorem 1 we get)

$$
\leq \prod_{i=1}^{m}\left(\int_{\Omega_{2}} u_{i}(y) \Phi_{i}\left(\left|f_{i}(y)\right|\right)^{p_{i}} d \mu_{2}(y)\right)^{\frac{1}{p_{i}}} .
$$

proving the claim.

When $k(x, y):=k_{1}(x, y)=k_{2}(x, y)=\cdots=k_{m}(x, y)$, then $K(x):=$ $K_{1}(x)=K_{2}(x)=\cdots=K_{m}(x)$, we get by Theorems 2,3 the following:

Corollary 4. Assume that the function $x \mapsto\left(u(x) \frac{k(x, y)}{K(x)}\right)$ is integrable on $\Omega_{1}$, for each fixed $y \in \Omega_{2}$. Define $U$ on $\Omega_{2}$ by

$$
U(y):=\int_{\Omega_{1}} u(x) \frac{k(x, y)}{K(x)} d \mu_{1}(x)<\infty .
$$

Let $p, q>1: \frac{1}{p}+\frac{1}{q}=1$. Let the functions $\Phi_{1}, \Phi_{2}: \mathbb{R}_{+} \rightarrow \mathbb{R}_{+}$, be convex and increasing. Then

$$
\begin{gathered}
\int_{\Omega_{1}} u(x) \Phi_{1}\left(\left|\frac{g_{1}(x)}{K(x)}\right|\right) \Phi_{2}\left(\left|\frac{g_{2}(x)}{K(x)}\right|\right) d \mu_{1}(x) \\
\leq\left(\int_{\Omega_{2}} U(y) \Phi_{1}\left(\left|f_{1}(y)\right|\right)^{p} d \mu_{2}(y)\right)^{\frac{1}{p}}\left(\int_{\Omega_{2}} U(y) \Phi_{2}\left(\left|f_{2}(y)\right|\right)^{q} d \mu_{2}(y)\right)^{\frac{1}{q}},
\end{gathered}
$$

for all measurable functions $f_{i}: \Omega_{2} \rightarrow \mathbb{R}(i=1,2)$ such that

(i) $f_{1}, f_{2}, \Phi_{1}\left(\left|f_{1}\right|\right)^{p}, \Phi_{2}\left(\left|f_{2}\right|\right)^{q}$ are all $k(x, y) d \mu_{2}(y)$-integrable, $\mu_{1}$-a.e. in $x \in \Omega_{1}$, 
(ii) $U \Phi_{1}\left(\left|f_{1}\right|\right)^{p}, U \Phi_{2}\left(\left|f_{2}\right|\right)^{q}$, are both $\mu_{2}$-integrable, and for all corresponding functions $g_{i}(i=1,2)$ given by $(10)$.

Corollary 5. Assume that the function $x \mapsto\left(u(x) \frac{k(x, y)}{K(x)}\right)$ is integrable on $\Omega_{1}$, for each fixed $y \in \Omega_{2}$. Define $U$ on $\Omega_{2}$ by

$$
U(y):=\int_{\Omega_{1}} u(x) \frac{k(x, y)}{K(x)} d \mu_{1}(x)<\infty .
$$

Let $p_{i}>1: \sum_{i=1}^{m} \frac{1}{p_{i}}=1$. Let the functions $\Phi_{i}: \mathbb{R}_{+} \rightarrow \mathbb{R}_{+}, i=1, \ldots, m$, be convex and increasing. Then

$$
\begin{aligned}
& \int_{\Omega_{1}} u(x) \prod_{i=1}^{m} \Phi_{i}\left(\left|\frac{g_{i}(x)}{K(x)}\right|\right) d \mu_{1}(x) \\
& \leq \prod_{i=1}^{m}\left(\int_{\Omega_{2}} U(y) \Phi_{i}\left(\left|f_{i}(y)\right|\right)^{p_{i}} d \mu_{2}(y)\right)^{\frac{1}{p_{i}}},
\end{aligned}
$$

for all measurable functions $f_{i}: \Omega_{2} \rightarrow \mathbb{R}, i=1, \ldots, m$, such that

(i) $f_{i}, \Phi_{i}\left(\left|f_{i}\right|\right)^{p_{i}}$ are both $k(x, y) d \mu_{2}(y)$-integrable, $\mu_{1}$-a.e. in $x \in \Omega_{1}$, for all $i=1, \ldots, m$,

(ii) $U \Phi_{i}\left(\left|f_{i}\right|\right)^{p_{i}}$ is $\mu_{2}$-integrable, $i=1, \ldots, m$,

and for all corresponding functions $g_{i}(i=1, \ldots, m)$ given by (10).

Next we give two applications of Theorem 3 .

Theorem 6. Assume that the functions $(i=1,2, \ldots, m \in \mathbb{N}) x \mapsto(u(x)$ $\left.\cdot \frac{k_{i}(x, y)}{K_{i}(x)}\right)$ are integrable on $\Omega_{1}$, for each fixed $y \in \Omega_{2}$. Define $u_{i}$ on $\Omega_{2}$ by

$$
u_{i}(y):=\int_{\Omega_{1}} u(x) \frac{k_{i}(x, y)}{K_{i}(x)} d \mu_{1}(x)<\infty .
$$

Let $p_{i}>1: \sum_{i=1}^{m} \frac{1}{p_{i}}=1 ; \alpha_{i} \geq 1, i=1, \ldots, m$.

Then

$$
\begin{aligned}
& \int_{\Omega_{1}} u(x)\left(\prod_{i=1}^{m}\left|\frac{g_{i}(x)}{K_{i}(x)}\right|^{\alpha_{i}}\right) d \mu_{1}(x) \\
& \leq \prod_{i=1}^{m}\left(\int_{\Omega_{2}} u_{i}(y)\left|f_{i}(y)\right|^{\alpha_{i} p_{i}} d \mu_{2}(y)\right)^{\frac{1}{p_{i}}}
\end{aligned}
$$

for all measurable functions $f_{i}: \Omega_{2} \rightarrow \mathbb{R}, i=1, \ldots, m$, such that

(i) $f_{i},\left|f_{i}\right|^{\alpha_{i} p_{i}}$ are $k_{i}(x, y) d \mu_{2}(y)$-integrable, $\mu_{1}$-a.e. in $x \in \Omega_{1}, i=$ $1, \ldots, m$

(ii) $u_{i}\left|f_{i}\right|^{\alpha_{i} p_{i}}$ is $\mu_{2}$-integrable, $i=1, \ldots, m$, 
and for all corresponding functions $g_{i}(i=1, \ldots, m)$ given by (10).

Theorem 7. Assume that the functions $(i=1,2, \ldots, m \in \mathbb{N}) x \mapsto(u(x)$ $\left.\cdot \frac{k_{i}(x, y)}{K_{i}(x)}\right)$ are integrable on $\Omega_{1}$, for each fixed $y \in \Omega_{2}$. Define $u_{i}$ on $\Omega_{2}$ by

$$
u_{i}(y):=\int_{\Omega_{1}} u(x) \frac{k_{i}(x, y)}{K_{i}(x)} d \mu_{1}(x)<\infty .
$$

Let $p_{i}>1: \sum_{i=1}^{m} \frac{1}{p_{i}}=1$. Then

$$
\begin{aligned}
& \int_{\Omega_{1}} u(x)\left(e^{\sum_{i=1}^{m}\left|\frac{g_{i}(x)}{K_{i}(x)}\right|}\right) d \mu_{1}(x) \\
& \leq \prod_{i=1}^{m}\left(\int_{\Omega_{2}} u_{i}(y) e^{p_{i}\left|f_{i}(y)\right|} d \mu_{2}(y)\right)^{\frac{1}{p_{i}}},
\end{aligned}
$$

for all measurable functions $f_{i}: \Omega_{2} \rightarrow \mathbb{R}, i=1, \ldots, m$, such that

(i) $f_{i}, e^{p_{i}\left|f_{i}\right|}$ are $k_{i}(x, y) d \mu_{2}(y)$-integrable, $\mu_{1}$-a.e. in $x \in \Omega_{1}, i=$ $1, \ldots, m$

(ii) $u_{i} e^{p_{i}\left|f_{i}\right|}$ is $\mu_{2}$-integrable, $i=1, \ldots, m$,

and for all corresponding functions $g_{i}(i=1, \ldots, m)$ given by $(10)$.

We need

Definition 8. ([16]) Let $(a, b), 0 \leq a<b<\infty ; \alpha, \sigma>0$. We consider the left- and right-sided fractional integrals of order $\alpha$ as follows:

1) for $\eta>-1$, we define

$$
\left(I_{a+; \sigma, \eta}^{\alpha} f\right)(x)=\frac{\sigma x^{-\sigma(\alpha+\eta)}}{\Gamma(\alpha)} \int_{a}^{x} \frac{t^{\sigma \eta+\sigma-1} f(t) d t}{\left(x^{\sigma}-t^{\sigma}\right)^{1-\alpha}},
$$

2) for $\eta>0$, we define

$$
\left(I_{b-; \sigma, \eta}^{\alpha} f\right)(x)=\frac{\sigma x^{\sigma \eta}}{\Gamma(\alpha)} \int_{x}^{b} \frac{t^{\sigma(1-\eta-\alpha)-1} f(t) d t}{\left(t^{\sigma}-x^{\sigma}\right)^{1-\alpha}} .
$$

These are the Erdélyi-Kober type fractional integrals.

We remind the Beta function

$$
B(x, y):=\int_{0}^{1} t^{x-1}(1-t)^{y-1} d t
$$

for $\Re(x), \Re(y)>0$, and the Incomplete Beta function

$$
B(x ; \alpha, \beta)=\int_{0}^{x} t^{\alpha-1}(1-t)^{\beta-1} d t,
$$

where $0<x \leq 1 ; \alpha, \beta>0$.

We make 
Remark 9. Regarding (27) we have

$$
k(x, y)=\frac{\sigma x^{-\sigma(\alpha+\eta)}}{\Gamma(\alpha)} \chi_{(a, x]}(y) \frac{y^{\sigma \eta+\sigma-1}}{\left(x^{\sigma}-y^{\sigma}\right)^{1-\alpha}},
$$

$x, y \in(a, b), \chi$ stands for the characteristic function.

Here

$$
K(x)=\int_{a}^{b} k(x, t) d t=\left(I_{a+; \sigma ; \eta}^{\alpha} 1\right)(x)=\frac{\sigma x^{-\sigma(\alpha+\eta)}}{\Gamma(\alpha)} \int_{a}^{x} \frac{t^{\sigma \eta+\sigma-1}}{\left(x^{\sigma}-t^{\sigma}\right)^{1-\alpha}} d t
$$

(setting $z=\frac{t}{x}$ )

$$
=\frac{\sigma}{\Gamma(\alpha)} \int_{\frac{a}{x}}^{1} z^{\sigma\left((\eta+1)-\frac{1}{\sigma}\right)}\left(1-z^{\sigma}\right)^{\alpha-1} d z
$$

(setting $\lambda=z^{\sigma}$ )

$$
=\frac{1}{\Gamma(\alpha)} \int_{\left(\frac{a}{x}\right)^{\sigma}}^{1} \lambda^{\eta}(1-\lambda)^{\alpha-1} d \lambda .
$$

Hence

$$
K(x)=\frac{1}{\Gamma(\alpha)} \int_{\left(\frac{a}{x}\right)^{\sigma}}^{1} \lambda^{\eta}(1-\lambda)^{\alpha-1} d \lambda .
$$

Indeed it is

$$
K(x)=\left(I_{a+; \sigma ; \eta}^{\alpha}(1)\right)(x)=\frac{B(\eta+1, \alpha)-B\left(\left(\frac{a}{x}\right)^{\sigma} ; \eta+1, \alpha\right)}{\Gamma(\alpha)} .
$$

We also make

Remark 10. Regarding (28) we have

$$
k(x, y)=\frac{\sigma x^{\sigma \eta}}{\Gamma(\alpha)} \chi_{[x, b)}(y) \frac{y^{\sigma(1-\eta-\alpha)-1}}{\left(y^{\sigma}-x^{\sigma}\right)^{1-\alpha}},
$$

$x, y \in(a, b)$. Here

$$
K(x)=\int_{a}^{b} k(x, t) d t=\left(I_{b-; \sigma ; \eta}^{\alpha} 1\right)(x)=\frac{\sigma x^{\sigma \eta}}{\Gamma(\alpha)} \int_{x}^{b} \frac{t^{\sigma(1-\eta-\alpha)-1}}{\left(t^{\sigma}-x^{\sigma}\right)^{1-\alpha}} d t
$$

(setting $z=\frac{t}{x}$ )

$$
\begin{aligned}
= & \frac{\sigma}{\Gamma(\alpha)} \int_{1}^{\left(\frac{b}{x}\right)}\left(z^{\sigma}-1\right)^{\alpha-1} z^{\sigma(1-\eta-\alpha)-1} d z \\
\left(\operatorname{setting} \lambda=z^{\sigma}, 1 \leq\right. & \left.\lambda<\left(\frac{b}{x}\right)^{\sigma}\right) \\
& =\frac{1}{\Gamma(\alpha)} \int_{1}^{\left(\frac{b}{x}\right)^{\sigma}}(\lambda-1)^{\alpha-1} \lambda^{-\eta-\alpha} d \lambda
\end{aligned}
$$




$$
=\frac{1}{\Gamma(\alpha)} \int_{1}^{\left(\frac{b}{x}\right)^{\sigma}} \frac{1}{\lambda^{\eta+1}}\left(1-\frac{1}{\lambda}\right)^{\alpha-1} d \lambda
$$

(setting $w:=\frac{1}{\lambda}, 0<\left(\frac{x}{b}\right)^{\sigma}<w \leq 1$ )

$$
=\frac{1}{\Gamma(\alpha)} \int_{\left(\frac{x}{b}\right)^{\sigma}}^{1} w^{\eta-1}(1-w)^{\alpha-1} d w=\frac{\left(B(\eta, \alpha)-B\left(\left(\frac{x}{b}\right)^{\sigma} ; \eta, \alpha\right)\right)}{\Gamma(\alpha)} .
$$

That is

$$
K(x)=\left(I_{b-; \sigma ; \eta}^{\alpha}(1)\right)(x)=\frac{\left(B(\eta, \alpha)-B\left(\left(\frac{x}{b}\right)^{\sigma} ; \eta, \alpha\right)\right)}{\Gamma(\alpha)} .
$$

We give

Theorem 11. Assume that the function

$$
x \mapsto\left(u(x) \frac{\chi_{(a, x]}(y) \sigma x^{-\sigma(\alpha+\eta)} y^{\sigma \eta+\sigma-1}}{\left(x^{\sigma}-y^{\sigma}\right)^{1-\alpha}\left[B(\eta+1, \alpha)-B\left(\left(\frac{a}{x}\right)^{\sigma} ; \eta+1, \alpha\right)\right]}\right)
$$

is integrable on $(a, b)$, for each $y \in(a, b)$. Here $\alpha, \sigma>0, \eta>-1,0 \leq a<$ $b<\infty$. Define $u_{1}$ on $(a, b)$ by

$$
u_{1}(y):=\sigma y^{\sigma \eta+\sigma-1} \int_{y}^{b} \frac{u(x) x^{-\sigma(\alpha+\eta)}\left(x^{\sigma}-y^{\sigma}\right)^{\alpha-1}}{\left(B(\eta+1, \alpha)-B\left(\left(\frac{a}{x}\right)^{\sigma} ; \eta+1, \alpha\right)\right)} d x<\infty .
$$

Let $p_{i}>1: \sum_{i=1}^{m} \frac{1}{p_{i}}=1$. Let the functions $\Phi_{i}: \mathbb{R}_{+} \rightarrow \mathbb{R}_{+}, i=1, \ldots, m$, be convex and increasing.

Then

$$
\begin{gathered}
\int_{a}^{b} u(x) \prod_{i=1}^{m} \Phi_{i}\left(\frac{\left|I_{a+; \sigma ; \eta}^{\alpha} f_{i}(x)\right| \Gamma(\alpha)}{\left(B(\eta+1, \alpha)-B\left(\left(\frac{a}{x}\right)^{\sigma} ; \eta+1, \alpha\right)\right)}\right) d x \\
\leq \prod_{i=1}^{m}\left(\int_{a}^{b} u_{1}(y) \Phi_{i}\left(\left|f_{i}(y)\right|\right)^{p_{i}} d y\right)^{\frac{1}{p_{i}}},
\end{gathered}
$$

for all measurable functions $f_{i}:(a, b) \rightarrow \mathbb{R}, i=1, \ldots, m$, such that

(i) $f_{i}, \Phi_{i}\left(\left|f_{i}\right|\right)^{p_{i}}$ are both $\frac{\sigma x^{-\sigma(\alpha+\eta)}}{\Gamma(\alpha)} \chi_{(a, x]}(y) \frac{y^{\sigma \eta+\sigma-1} d y}{\left(x^{\sigma}-y^{\sigma}\right)^{1-\alpha}}$-integrable, a.e. in $x \in(a, b)$, for all $i=1, \ldots, m$,

(ii) $u_{1} \Phi_{i}\left(\left|f_{i}\right|\right)^{p_{i}}$ is Lebesgue integrable, $i=1, \ldots, m$,

Proof. By Corollary 5.

Remark 12. In (42), if we choose

$$
u(x)=x^{\sigma(\alpha+\eta+1)-1}\left(B(\eta+1, \alpha)-B\left(\left(\frac{a}{x}\right)^{\alpha} ; \eta+1, \alpha\right)\right), x \in(a, b),
$$


then

$$
u_{1}(y)=\sigma y^{\sigma \eta+\sigma-1} \int_{y}^{b} x^{\sigma-1}\left(x^{\sigma}-y^{\sigma}\right)^{\alpha-1} d x
$$

$\left(\operatorname{setting} w:=x^{\sigma}, \frac{d w}{d x}=\sigma x^{\sigma-1}, d x=\frac{d w}{\sigma x^{\sigma-1}}\right)$

$$
=y^{\sigma \eta+\sigma-1} \int_{y^{\sigma}}^{b^{\sigma}}\left(w-y^{\sigma}\right)^{\alpha-1} d w=y^{\sigma \eta+\sigma-1} \frac{\left(b^{\sigma}-y^{\sigma}\right)^{\alpha}}{\alpha} .
$$

That is

$$
u_{1}(y)=y^{\sigma \eta+\sigma-1} \frac{\left(b^{\sigma}-y^{\sigma}\right)^{\alpha}}{\alpha}, y \in(a, b) .
$$

Based on the above, (43) becomes

$$
\begin{aligned}
& \int_{a}^{b} x^{\sigma(\alpha+\eta+1)-1}\left(B(\eta+1, \alpha)-B\left(\left(\frac{a}{x}\right)^{\sigma} ; \eta+1, \alpha\right)\right) \\
& \quad \prod_{i=1}^{m} \Phi_{i}\left(\frac{\left|I_{a+; \sigma ; \eta}^{\alpha} f_{i}(x)\right| \Gamma(\alpha)}{\left(B(\eta+1, \alpha)-B\left(\left(\frac{a}{x}\right)^{\sigma} ; \eta+1, \alpha\right)\right)}\right) d x \\
& \leq \frac{1}{\alpha} \prod_{i=1}^{m}\left(\int_{a}^{b} y^{\sigma \eta+\sigma-1}\left(b^{\sigma}-y^{\sigma}\right)^{\alpha} \Phi_{i}\left(\left|f_{i}(y)\right|\right)^{p_{i}} d y\right)^{\frac{1}{p_{i}}} \\
& \leq \frac{\left(b^{\sigma}-a^{\sigma}\right)^{\alpha}}{\alpha} \prod_{i=1}^{m}\left(\int_{a}^{b} y^{\sigma(\eta+1)-1} \Phi_{i}\left(\left|f_{i}(y)\right|\right)^{p_{i}} d y\right)^{\frac{1}{p_{i}}}
\end{aligned}
$$

under the assumptions:

(i) following (43), and

(ii) ${ }^{*} y^{\sigma(\eta+1)-1} \Phi_{i}\left(\left|f_{i}(y)\right|\right)^{p_{i}}$ is Lebesgue integrable on $(a, b), i=1, \ldots, m$.

Corollary 13. Let $0 \leq a<b ; \alpha, \sigma>0, \eta>-1 ; p_{i}>1: \sum_{i=1}^{m} \frac{1}{p_{i}}=1$; $\beta_{i} \geq 1, i=1, \ldots, m$. Then

$$
\begin{gathered}
\int_{a}^{b} x^{\sigma(\alpha+\eta+1)-1}\left(B(\eta+1, \alpha)-B\left(\left(\frac{a}{x}\right)^{\sigma} ; \eta+1, \alpha\right)\right)^{\left(1-\sum_{i=1}^{m} \beta_{i}\right)} \\
\cdot\left(\prod_{i=1}^{m}\left|I_{a+; \sigma ; \eta}^{\alpha} f_{i}(x)\right|^{\beta_{i}}\right) d x \leq \frac{1}{\alpha(\Gamma(\alpha))^{\sum_{i=1}^{m} \beta_{i}}} \\
\cdot \prod_{i=1}^{m}\left(\int_{a}^{b} y^{\sigma \eta+\sigma-1}\left(b^{\sigma}-y^{\sigma}\right)^{\alpha}\left|f_{i}(y)\right|^{\beta_{i} p_{i}} d y\right)^{\frac{1}{p_{i}}} \\
\leq\left(\frac{\left(b^{\sigma}-a^{\sigma}\right)^{\alpha}}{\alpha(\Gamma(\alpha))^{\sum_{i=1}^{m} \beta_{i}}}\right) \prod_{i=1}^{m}\left(\int_{a}^{b} y^{\sigma(\eta+1)-1}\left|f_{i}(y)\right|^{\beta_{i} p_{i}} d y\right)^{\frac{1}{p_{i}}}
\end{gathered}
$$

for all measurable functions $f_{i}:(a, b) \rightarrow \mathbb{R}, i=1, \ldots, m$ such that 
(i) $\left|f_{i}\right|^{\beta_{i} p_{i}}$ is $\left(\frac{\sigma x^{-\sigma(\alpha+\eta)}}{\Gamma(\alpha)} \chi_{(a, x]}(y) \frac{y^{\sigma \eta+\sigma-1} d y}{\left(x^{\sigma}-y^{\sigma}\right)^{1-\alpha}}\right)$-integrable, a.e. $\quad$ in $x \in$ $(a, b)$

(ii) $y^{\sigma(\eta+1)-1}\left|f_{i}(y)\right|^{\beta_{i} p_{i}}$ is Lebesgue integrable on $(a, b) ; i=1, \ldots, m$.

Proof. By Theorem 11 and (47).

Corollary 14. Let $0 \leq a<b ; \alpha, \sigma>0, \eta>-1 ; p_{i}>1: \sum_{i=1}^{m} \frac{1}{p_{i}}=1$. Then

$$
\begin{gathered}
\int_{a}^{b} x^{\sigma(\alpha+\eta+1)-1}\left(B(\eta+1, \alpha)-B\left(\left(\frac{a}{x}\right)^{\sigma} ; \eta+1, \alpha\right)\right) \\
\quad \cdot e^{\frac{\Gamma(\alpha)\left(\sum_{i=1}^{m}\left|I_{a+; \sigma ; \eta}^{\alpha} f_{i}(x)\right|\right)}{\left(B(\eta+1, \alpha)-B\left(\left(\frac{a}{x}\right)^{\sigma} ; \eta+1, \alpha\right)\right)}} d x \\
\leq \frac{1}{\alpha} \prod_{i=1}^{m}\left(\int_{a}^{b} y^{\sigma(\eta+1)-1}\left(b^{\sigma}-y^{\sigma}\right)^{\alpha} e^{p_{i}\left|f_{i}(y)\right|} d y\right)^{\frac{1}{p_{i}}} \\
\leq \frac{\left(b^{\sigma}-a^{\sigma}\right)^{\alpha}}{\alpha} \prod_{i=1}^{m}\left(\int_{a}^{b} y^{\sigma(\eta+1)-1} e^{p_{i}\left|f_{i}(y)\right|} d y\right)^{\frac{1}{p_{i}}}
\end{gathered}
$$

for all measurable functions $f_{i}:(a, b) \rightarrow \mathbb{R}, i=1, \ldots, m$ such that

(i) $f_{i}, e^{p_{i}\left|f_{i}\right|}$ are both $\frac{\sigma x^{-\sigma(\alpha+\eta)}}{\Gamma(\alpha)} \chi_{(a, x]}(y) \frac{y^{\sigma \eta+\sigma-1} d y}{\left(x^{\sigma}-y^{\sigma}\right)^{1-\alpha}}$ integrable, a.e. in $x \in(a, b)$,

(ii) $y^{\sigma(\eta+1)-1} e^{p_{i}\left|f_{i}(y)\right|}$ is Lebesgue integrable on $(a, b) ; i=1, \ldots, m$.

Proof. By Theorem 11 and (47).

We present

Theorem 15. Assume that the function

$$
x \mapsto\left(u(x) \frac{\sigma x^{\sigma \eta} \chi_{[x, b)}(y) y^{\sigma(1-\eta-\alpha)-1}}{\left(y^{\sigma}-x^{\sigma}\right)^{1-\alpha}\left[B(\eta, \alpha)-B\left(\left(\frac{x}{b}\right)^{\sigma} ; \eta, \alpha\right)\right]}\right)
$$

is integrable on $(a, b)$, for each $y \in(a, b)$. Here $\alpha, \sigma, \eta>0,0 \leq a<b<\infty$. Define $u_{2}$ on $(a, b)$ by

$$
u_{2}(y):=\sigma y^{\sigma(1-\eta-\alpha)-1} \int_{a}^{y} \frac{u(x) x^{\sigma \eta}\left(y^{\sigma}-x^{\sigma}\right)^{\alpha-1} d x}{\left(B(\eta, \alpha)-B\left(\left(\frac{x}{b}\right)^{\sigma} ; \eta, \alpha\right)\right)}<\infty .
$$

Let $p_{i}>1: \sum_{i=1}^{m} \frac{1}{p_{i}}=1$. Let the functions $\Phi_{i}: \mathbb{R}_{+} \rightarrow \mathbb{R}_{+}, i=1, \ldots, m$, be convex and increasing. Then

$$
\int_{a}^{b} u(x) \prod_{i=1}^{m} \Phi_{i}\left(\frac{\left|I_{b-; \sigma ; \eta}^{\alpha} f_{i}(x)\right| \Gamma(\alpha)}{\left(B(\eta, \alpha)-B\left(\left(\frac{x}{b}\right)^{\sigma} ; \eta, \alpha\right)\right)}\right) d x
$$




$$
\leq \prod_{i=1}^{m}\left(\int_{a}^{b} u_{2}(y) \Phi_{i}\left(\left|f_{i}(y)\right|\right)^{p_{i}} d y\right)^{\frac{1}{p_{i}}},
$$

for all measurable functions $f_{i}:(a, b) \rightarrow \mathbb{R}, i=1, \ldots, m$, such that

(i) $f_{i}, \Phi_{i}\left(\left|f_{i}\right|\right)^{p_{i}}$ are both $\left(\frac{\sigma x^{\sigma \eta} \chi_{[x, b)}(y) y^{\sigma(1-\eta-\alpha)-1} d y}{\Gamma(\alpha)\left(y^{\sigma}-x^{\sigma}\right)^{1-\alpha}}\right)$-integrable, a.e. in $x \in(a, b)$, for all $i=1, \ldots, m$,

(ii) $u_{2} \Phi_{i}\left(\left|f_{i}\right|\right)^{p_{i}}$ is Lebesgue integrable on $(a, b), i=1, \ldots, m$.

Proof. By Corollary 5.

Remark 16. Here $0<a<b<\infty ; \alpha, \sigma, \eta>0$.

In (50), if we choose

$$
u(x)=x^{\sigma(1-\eta)-1}\left(B(\eta, \alpha)-B\left(\left(\frac{x}{b}\right)^{\alpha} ; \eta, \alpha\right)\right), x \in(a, b),
$$

then

$$
u_{2}(y)=\sigma y^{\sigma(1-\eta-\alpha)-1} \int_{a}^{y} x^{\sigma-1}\left(y^{\sigma}-x^{\sigma}\right)^{\alpha-1} d x
$$

(setting $w:=x^{\sigma}, d x=\frac{d w}{\sigma x^{\sigma-1}}$ )

$$
=y^{\sigma(1-\eta-\alpha)-1} \int_{a^{\sigma}}^{y^{\sigma}}\left(y^{\sigma}-w\right)^{\alpha-1} d w=y^{\sigma(1-\eta-\alpha)-1} \frac{\left(y^{\sigma}-a^{\sigma}\right)^{\alpha}}{\alpha} .
$$

That is

$$
u_{2}(y)=y^{\sigma(1-\eta-\alpha)-1} \frac{\left(y^{\sigma}-a^{\sigma}\right)^{\alpha}}{\alpha}, y \in(a, b) .
$$

Based on the above, (51) becomes

$$
\begin{gathered}
\int_{a}^{b} x^{\sigma(1-\eta)-1}\left(B(\eta, \alpha)-B\left(\left(\frac{x}{b}\right)^{\sigma} ; \eta, \alpha\right)\right) \\
\cdot \prod_{i=1}^{m} \Phi_{i}\left(\frac{\left|I_{b-; \sigma ; \eta}^{\alpha} f_{i}(x)\right| \Gamma(\alpha)}{\left(B(\eta, \alpha)-B\left(\left(\frac{x}{b}\right)^{\sigma} ; \eta, \alpha\right)\right)}\right) d x \\
\leq \frac{1}{\alpha} \prod_{i=1}^{m}\left(\int_{a}^{b} y^{\sigma(1-\eta-\alpha)-1}\left(y^{\sigma}-a^{\sigma}\right)^{\alpha} \Phi_{i}\left(\left|f_{i}(y)\right|\right)^{p_{i}} d y\right)^{\frac{1}{p_{i}}} \\
\leq \frac{\left(b^{\sigma}-a^{\sigma}\right)^{\alpha}}{\alpha} \prod_{i=1}^{m}\left(\int_{a}^{b} y^{\alpha(1-\eta-\alpha)-1} \Phi_{i}\left(\left|f_{i}(y)\right|\right)^{p_{i}} d y\right)^{\frac{1}{p_{i}}},
\end{gathered}
$$

under the assumptions:

(i) following (51), and

(ii) $^{*} y^{\sigma(1-\eta-\alpha)-1} \Phi_{i}\left(\left|f_{i}(y)\right|\right)^{p_{i}}$ is Lebesgue integrable on $(a, b), i=1, \ldots, m$. 
Corollary 17. Let $0<a<b<\infty ; \alpha, \sigma, \eta>0 ; p_{i}>1: \sum_{i=1}^{m} \frac{1}{p_{i}}=1$; $\beta_{i} \geq 1, i=1, \ldots, m$. Then

$$
\begin{gathered}
\int_{a}^{b} x^{\sigma(1-\eta)-1}\left(B(\eta, \alpha)-B\left(\left(\frac{x}{b}\right)^{\sigma} ; \eta, \alpha\right)\right)^{\left(1-\sum_{i=1}^{m} \beta_{i}\right)} \\
\cdot\left(\prod_{i=1}^{m}\left|I_{b-; \sigma ; \eta}^{\alpha} f_{i}(x)\right|^{\beta_{i}}\right) d x \leq \frac{1}{\alpha(\Gamma(\alpha))^{\sum_{i=1}^{m} \beta_{i}}} \\
\cdot \prod_{i=1}^{m}\left(\int_{a}^{b} y^{\sigma(1-\eta-\alpha)-1}\left(y^{\sigma}-a^{\sigma}\right)^{\alpha}\left|f_{i}(y)\right|^{\beta_{i} p_{i}} d y\right)^{\frac{1}{p_{i}}} \\
\leq \frac{\left(b^{\sigma}-a^{\sigma}\right)^{\alpha}}{\alpha(\Gamma(\alpha))^{\sum_{i=1}^{m} \beta_{i}}} \prod_{i=1}^{m}\left(\int_{a}^{b} y^{\sigma(1-\eta-\alpha)-1}\left|f_{i}(y)\right|^{\beta_{i} p_{i}} d y\right)^{\frac{1}{p_{i}}}
\end{gathered}
$$

under the assumptions:

(i) $\left|f_{i}\right|^{\beta_{i} p_{i}}$ is $\left(\frac{\sigma x^{\sigma \eta} \chi_{[x, b)}(y) y^{\sigma(1-\eta-\alpha)-1} d y}{\Gamma(\alpha)\left(y^{\sigma}-x^{\sigma}\right)^{1-\alpha}}\right)$-integrable, a.e. in $x \in(a, b)$, for all $i=1, \ldots, m$,

(ii) $y^{\sigma(1-\eta-\alpha)-1}\left|f_{i}(y)\right|^{\beta_{i} p_{i}}$ is Lebesgue integrable on $(a, b), i=1, \ldots, m$.

Proof. By Theorem 15 and (55).

Corollary 18. Let $0<a<b<\infty ; \alpha, \sigma, \eta>0 ; p_{i}>1: \sum_{i=1}^{m} \frac{1}{p_{i}}=1$. Then

$$
\begin{gathered}
\int_{a}^{b} x^{\sigma(1-\eta)-1}\left(B(\eta, \alpha)-B\left(\left(\frac{x}{b}\right)^{\sigma} ; \eta, \alpha\right)\right) \cdot e^{\frac{\Gamma(\alpha)\left(\sum_{i=1}^{m}\left|I_{b-; \sigma ;}^{\alpha} f_{i}(x)\right|\right)}{\left(B(\eta, \alpha)-B\left(\left(\frac{x}{b}\right)^{\circ} ; \eta, \alpha\right)\right)}} d x \\
\leq \frac{1}{\alpha} \prod_{i=1}^{m}\left(\int_{a}^{b} y^{\sigma(1-\eta-\alpha)-1}\left(y^{\sigma}-a^{\sigma}\right)^{\alpha} e^{p_{i}\left|f_{i}(y)\right|} d y\right)^{\frac{1}{p_{i}}} \\
\leq \frac{\left(b^{\sigma}-a^{\sigma}\right)^{\alpha}}{\alpha} \prod_{i=1}^{m}\left(\int_{a}^{b} y^{\sigma(1-\eta-\alpha)-1} e^{p_{i}\left|f_{i}(y)\right|} d y\right)^{\frac{1}{p_{i}}},
\end{gathered}
$$

under the assumptions:

(i) $f_{i}, e^{p_{i}\left|f_{i}\right|}$ are both $\left(\frac{\sigma x^{\sigma \eta} \chi_{[x, b)}(y) y^{\sigma(1-\eta-\alpha)-1} d y}{\Gamma(\alpha)\left(y^{\sigma}-x^{\sigma}\right)^{1-\alpha}}\right)$-integrable, a.e. in $x \in$ $(a, b), i=1, \ldots, m$,

(ii) $y^{\sigma(1-\eta-\alpha)-1} e^{p_{i}\left|f_{i}(y)\right|}$ is Lebesgue integrable on $(a, b) ; i=1, \ldots, m$.

Proof. By Theorem 15 and (55).

We make 
Remark 19. Let $\prod_{i=1}^{N}\left(a_{i}, b_{i}\right) \subset \mathbb{R}^{N}, N>1, a_{i}<b_{i}, a_{i}, b_{i} \in \mathbb{R}$. Let $\alpha_{i}>0, i=1, \ldots, N ; f \in L_{1}\left(\prod_{i=1}^{N}\left(a_{i}, b_{i}\right)\right)$, and set $a=\left(a_{1}, \ldots, a_{N}\right)$, $b=\left(b_{1}, \ldots, b_{N}\right), \alpha=\left(\alpha_{1}, \ldots, \alpha_{N}\right), x=\left(x_{1}, \ldots, x_{N}\right), t=\left(t_{1}, \ldots, t_{N}\right)$.

We define the left mixed Riemann-Liouville fractional multiple integral of order $\alpha$ (see also [14]):

$\left(I_{a+}^{\alpha} f\right)(x):=\frac{1}{\prod_{i=1}^{N} \Gamma\left(\alpha_{i}\right)} \int_{a_{1}}^{x_{1}} \ldots \int_{a_{N}}^{x_{N}} \prod_{i=1}^{N}\left(x_{i}-t_{i}\right)^{\alpha_{i}-1} f\left(t_{1}, \ldots, t_{N}\right) d t_{1} \ldots d t_{N}$,

with $x_{i}>a_{i}, i=1, \ldots, N$.

We also define the right mixed Riemann-Liouville fractional multiple integral of order $\alpha$ (see also [12]):

$$
\left(I_{b-}^{\alpha} f\right)(x):=\frac{1}{\prod_{i=1}^{N} \Gamma\left(\alpha_{i}\right)} \int_{x_{1}}^{b_{1}} \ldots \int_{x_{N}}^{b_{N}} \prod_{i=1}^{N}\left(t_{i}-x_{i}\right)^{\alpha_{i}-1} f\left(t_{1}, \ldots, t_{N}\right) d t_{1} \ldots d t_{N},
$$

with $x_{i}<b_{i}, i=1, \ldots, N$.

Notice $I_{a+}^{\alpha}(|f|), I_{b-}^{\alpha}(|f|)$ are finite if $f \in L_{\infty}\left(\prod_{i=1}^{N}\left(a_{i}, b_{i}\right)\right)$.

One can rewrite (58) and (59) as follows:

$$
\left(I_{a+}^{\alpha} f\right)(x)=\frac{1}{\prod_{i=1}^{N} \Gamma\left(\alpha_{i}\right)} \int_{\prod_{i=1}^{N}\left(a_{i}, b_{i}\right)} \chi_{\prod_{i=1}^{N}\left(a_{i}, x_{i}\right]}(t) \prod_{i=1}^{N}\left(x_{i}-t_{i}\right)^{\alpha_{i}-1} f(t) d t,
$$

with $x_{i}>a_{i}, i=1, \ldots, N$, and

$$
\left(I_{b-}^{\alpha} f\right)(x)=\frac{1}{\prod_{i=1}^{N} \Gamma\left(\alpha_{i}\right)} \int_{\prod_{i=1}^{N}\left(a_{i}, b_{i}\right)} \chi_{\prod_{i=1}^{N}\left[x_{i}, b_{i}\right)}(t) \prod_{i=1}^{N}\left(t_{i}-x_{i}\right)^{\alpha_{i}-1} f(t) d t
$$

with $x_{i}<b_{i}, i=1, \ldots, N$.

The corresponding $k(x, y)$ for $I_{a+}^{\alpha}, I_{b-}^{\alpha}$ are

$$
k_{a+}(x, y)=\frac{1}{\prod_{i=1}^{N} \Gamma\left(\alpha_{i}\right)} \chi_{\prod_{i=1}^{N}\left(a_{i}, x_{i}\right]}(y) \prod_{i=1}^{N}\left(x_{i}-y_{i}\right)^{\alpha_{i}-1},
$$

$\forall x, y \in \prod_{i=1}^{N}\left(a_{i}, b_{i}\right)$, and

$$
k_{b-}(x, y)=\frac{1}{\prod_{i=1}^{N} \Gamma\left(\alpha_{i}\right)} \chi_{\prod_{i=1}^{N}\left[x_{i}, b_{i}\right)}(y) \prod_{i=1}^{N}\left(y_{i}-x_{i}\right)^{\alpha_{i}-1},
$$


$\forall x, y \in \prod_{i=1}^{N}\left(a_{i}, b_{i}\right)$.

The corresponding $K(x)$ for $I_{a+}^{\alpha}$ is:

$$
\begin{gathered}
K_{a+}(x)=\int_{\prod_{i=1}^{N}\left(a_{i}, b_{i}\right)} k_{a+}(x, y) d y=\left(I_{a+}^{\alpha} 1\right)(x) \\
=\frac{1}{\prod_{i=1}^{N} \Gamma\left(\alpha_{i}\right)} \int_{a_{1}}^{x_{1}} \ldots \int_{a_{N}}^{x_{N}} \prod_{i=1}^{N}\left(x_{i}-t_{i}\right)^{\alpha_{i}-1} d t_{1} \ldots d t_{N} \\
=\frac{1}{\prod_{i=1}^{N} \Gamma\left(\alpha_{i}\right)} \prod_{i=1}^{N} \int_{a_{i}}^{x_{i}}\left(x_{i}-t_{i}\right)^{\alpha_{i}-1} d t_{i}=\frac{1}{\prod_{i=1}^{N} \Gamma\left(\alpha_{i}\right)} \prod_{i=1}^{N} \frac{\left(x_{i}-a_{i}\right)^{\alpha_{i}}}{\alpha_{i}} \\
=\prod_{i=1}^{N}\left(\frac{\left(x_{i}-a_{i}\right)^{\alpha_{i}}}{\Gamma\left(\alpha_{i}+1\right)}\right)
\end{gathered}
$$

that is

$$
K_{a+}(x)=\prod_{i=1}^{N} \frac{\left(x_{i}-a_{i}\right)^{\alpha_{i}}}{\Gamma\left(\alpha_{i}+1\right)},
$$

$\forall x \in \prod_{i=1}^{N}\left(a_{i}, b_{i}\right)$.

Similarly the corresponding $K(x)$ for $I_{b-}^{\alpha}$ is:

$$
\begin{gathered}
K_{b-}(x)=\int_{\prod_{i=1}^{N}\left(a_{i}, b_{i}\right)} k_{b-}(x, y) d y=\left(I_{b-}^{\alpha} 1\right)(x)= \\
\frac{1}{\prod_{i=1}^{N} \Gamma\left(\alpha_{i}\right)} \int_{x_{1}}^{b_{1}} \ldots \int_{x_{N}}^{b_{N}} \prod_{i=1}^{N}\left(t_{i}-x_{i}\right)^{\alpha_{i}-1} d t_{1} \ldots d t_{N}= \\
\frac{1}{\prod_{i=1}^{N} \Gamma\left(\alpha_{i}\right)} \prod_{i=1}^{N} \int_{x_{i}}^{b_{i}}\left(t_{i}-x_{i}\right)^{\alpha_{i}-1} d t_{i}=\frac{1}{\prod_{i=1}^{N} \Gamma\left(\alpha_{i}\right)} \prod_{i=1}^{N} \frac{\left(b_{i}-x_{i}\right)^{\alpha_{i}}}{\alpha_{i}} \\
=\prod_{i=1}^{N} \frac{\left(b_{i}-x_{i}\right)^{\alpha_{i}}}{\Gamma\left(\alpha_{i}+1\right)},
\end{gathered}
$$

that is

$$
K_{b-}(x)=\prod_{i=1}^{N} \frac{\left(b_{i}-x_{i}\right)^{\alpha_{i}}}{\Gamma\left(\alpha_{i}+1\right)},
$$


$\forall x \in \prod_{i=1}^{N}\left(a_{i}, b_{i}\right)$.

Next we form

$$
\begin{aligned}
\frac{k_{a+}(x, y)}{K_{a+}(x)} & =\frac{1}{\prod_{i=1}^{N} \Gamma\left(\alpha_{i}\right)} \chi_{\prod_{i=1}^{N}\left(a_{i}, x_{i}\right]}(y) \prod_{i=1}^{N}\left(x_{i}-y_{i}\right)^{\alpha_{i}-1} \prod_{i=1}^{N} \frac{\Gamma\left(\alpha_{i}+1\right)}{\left(x_{i}-a_{i}\right)^{\alpha_{i}}} \\
& =\chi_{\prod_{i=1}^{N}\left(a_{i}, x_{i}\right]}(y)\left(\prod_{i=1}^{N} \alpha_{i}\right)\left(\prod_{i=1}^{N} \frac{\left(x_{i}-y_{i}\right)^{\alpha_{i}-1}}{\left(x_{i}-a_{i}\right)^{\alpha_{i}}}\right),
\end{aligned}
$$

that is

$$
\frac{k_{a+}(x, y)}{K_{a+}(x)}=\chi_{\prod_{i=1}^{N}\left(a_{i}, x_{i}\right]}(y)\left(\prod_{i=1}^{N} \alpha_{i}\right)\left(\prod_{i=1}^{N} \frac{\left(x_{i}-y_{i}\right)^{\alpha_{i}-1}}{\left(x_{i}-a_{i}\right)^{\alpha_{i}}}\right)
$$

$\forall x, y \in \prod_{i=1}^{N}\left(a_{i}, b_{i}\right)$.

Similarly we form

$$
\begin{aligned}
\frac{k_{b-}(x, y)}{K_{b-}(x)} & =\frac{1}{\prod_{i=1}^{N} \Gamma\left(\alpha_{i}\right)} \chi_{\prod_{i=1}^{N}\left[x_{i}, b_{i}\right)}(y) \prod_{i=1}^{N}\left(y_{i}-x_{i}\right)^{\alpha_{i}-1} \prod_{i=1}^{N} \frac{\Gamma\left(\alpha_{i}+1\right)}{\left(b_{i}-x_{i}\right)^{\alpha_{i}}} \\
& =\chi_{\prod_{i=1}^{N}\left[x_{i}, b_{i}\right)}(y)\left(\prod_{i=1}^{N} \alpha_{i}\right)\left(\prod_{i=1}^{N} \frac{\left(y_{i}-x_{i}\right)^{\alpha_{i}-1}}{\left(b_{i}-x_{i}\right)^{\alpha_{i}}}\right),
\end{aligned}
$$

that is

$$
\frac{k_{b-}(x, y)}{K_{b-}(x)}=\chi_{\prod_{i=1}^{N}\left[x_{i}, b_{i}\right)}(y)\left(\prod_{i=1}^{N} \alpha_{i}\right)\left(\prod_{i=1}^{N} \frac{\left(y_{i}-x_{i}\right)^{\alpha_{i}-1}}{\left(b_{i}-x_{i}\right)^{\alpha_{i}}}\right)
$$

$\forall x, y \in \prod_{i=1}^{N}\left(a_{i}, b_{i}\right)$.

We choose the weight function $u_{1}(x)$ on $\prod_{i=1}^{N}\left(a_{i}, b_{i}\right)$ such that the function $x \mapsto\left(u_{1}(x) \frac{k_{a+}(x, y)}{K_{a+}(x)}\right)$ is integrable on $\prod_{i=1}^{N}\left(a_{i}, b_{i}\right)$, for each fixed $y \in$ $\prod_{i=1}^{N}\left(a_{i}, b_{i}\right)$. We define $w_{1}$ on $\prod_{i=1}^{N}\left(a_{i}, b_{i}\right)$ by

$$
w_{1}(y):=\int_{\prod_{i=1}^{N}\left(a_{i}, b_{i}\right)} u_{1}(x) \frac{k_{a+}(x, y)}{K_{a+}(x)} d x<\infty .
$$


We have that

$w_{1}(y)=\left(\prod_{i=1}^{N} \alpha_{i}\right) \int_{y_{1}}^{b_{1}} \ldots \int_{y_{N}}^{b_{N}} u_{1}\left(x_{1}, \ldots, x_{N}\right)\left(\prod_{i=1}^{N} \frac{\left(x_{i}-y_{i}\right)^{\alpha_{i}-1}}{\left(x_{i}-a_{i}\right)^{\alpha_{i}}}\right) d x_{1} \ldots d x_{N}$,

$\forall y \in \prod_{i=1}^{N}\left(a_{i}, b_{i}\right)$.

We also choose the weight function $u_{2}(x)$ on $\prod_{i=1}^{N}\left(a_{i}, b_{i}\right)$ such that the function $x \mapsto\left(u_{2}(x) \frac{k_{b-}(x, y)}{K_{b-}(x)}\right)$ is integrable on $\prod_{i=1}^{N}\left(a_{i}, b_{i}\right)$, for each fixed $y \in \prod_{i=1}^{N}\left(a_{i}, b_{i}\right)$. We define $w_{2}$ on $\prod_{i=1}^{N}\left(a_{i}, b_{i}\right)$ by

$$
w_{2}(y):=\int_{\prod_{i=1}^{N}\left(a_{i}, b_{i}\right)} u_{2}(x) \frac{k_{b-}(x, y)}{K_{b-}(x)} d x<\infty .
$$

We have that

$w_{2}(y)=\left(\prod_{i=1}^{N} \alpha_{i}\right) \int_{a_{1}}^{y_{1}} \ldots \int_{a_{N}}^{y_{N}} u_{2}\left(x_{1}, \ldots, x_{N}\right)\left(\prod_{i=1}^{N} \frac{\left(y_{i}-x_{i}\right)^{\alpha_{i}-1}}{\left(b_{i}-x_{i}\right)^{\alpha_{i}}}\right) d x_{1} \ldots d x_{N}$,

$\forall y \in \prod_{i=1}^{N}\left(a_{i}, b_{i}\right)$.

If we choose as

$$
u_{1}(x)=u_{1}^{*}(x):=\prod_{i=1}^{N}\left(x_{i}-a_{i}\right)^{\alpha_{i}}
$$

then

$$
\begin{gathered}
w_{1}^{*}(y):=w_{1}(y)=\left(\prod_{i=1}^{N} \alpha_{i}\right) \int_{y_{1}}^{b_{1}} \ldots \int_{y_{N}}^{b_{N}}\left(\prod_{i=1}^{N}\left(x_{i}-y_{i}\right)^{\alpha_{i}-1}\right) d x_{1} \ldots d x_{N} \\
=\left(\prod_{i=1}^{N} \alpha_{i}\right)\left(\prod_{i=1}^{N} \int_{y_{i}}^{b_{i}}\left(x_{i}-y_{i}\right)^{\alpha_{i}-1} d x_{i}\right) \\
=\left(\prod_{i=1}^{N} \alpha_{i}\right)\left(\prod_{i=1}^{N} \frac{\left(b_{i}-y_{i}\right)^{\alpha_{i}}}{\alpha_{i}}\right)=\prod_{i=1}^{N}\left(b_{i}-y_{i}\right)^{\alpha_{i}} .
\end{gathered}
$$

that is

$$
w_{1}^{*}(y)=\prod_{i=1}^{N}\left(b_{i}-y_{i}\right)^{\alpha_{i}}, \quad \forall y \in \prod_{i=1}^{N}\left(a_{i}, b_{i}\right) .
$$

If we choose as

$$
u_{2}(x)=u_{2}^{*}(x):=\prod_{i=1}^{N}\left(b_{i}-x_{i}\right)^{\alpha_{i}}
$$


then

$$
\begin{gathered}
w_{2}^{*}(y):=w_{2}(y)=\left(\prod_{i=1}^{N} \alpha_{i}\right) \int_{a_{1}}^{y_{1}} \ldots \int_{a_{N}}^{y_{N}}\left(\prod_{i=1}^{N}\left(y_{i}-x_{i}\right)^{\alpha_{i}-1}\right) d x_{1} \ldots d x_{N} \\
=\left(\prod_{i=1}^{N} \alpha_{i}\right)\left(\prod_{i=1}^{N} \int_{a_{i}}^{y_{i}}\left(y_{i}-x_{i}\right)^{\alpha_{i}-1} d x_{i}\right) \\
=\left(\prod_{i=1}^{N} \alpha_{i}\right)\left(\prod_{i=1}^{N} \frac{\left(y_{i}-a_{i}\right)^{\alpha_{i}}}{\alpha_{i}}\right)=\prod_{i=1}^{N}\left(y_{i}-a_{i}\right)^{\alpha_{i}} .
\end{gathered}
$$

That is

$$
w_{2}^{*}(y)=\prod_{i=1}^{N}\left(y_{i}-a_{i}\right)^{\alpha_{i}}, \quad \forall y \in \prod_{i=1}^{N}\left(a_{i}, b_{i}\right) .
$$

Here we choose $f_{j}: \prod_{i=1}^{N}\left(a_{i}, b_{i}\right) \rightarrow \mathbb{R}, j=1, \ldots, m$, that are Lebesgue measurable and $I_{a+}^{\alpha}\left(\left|f_{j}\right|\right), I_{b-}^{\alpha}\left(\left|f_{j}\right|\right)$ are finite a.e., one or the other, or both.

Let $p_{j}>1: \sum_{j=1}^{m} \frac{1}{p_{j}}=1$ and the functions $\Phi_{j}: \mathbb{R}_{+} \rightarrow \mathbb{R}_{+}, j=1, \ldots, m$, to be convex and increasing.

Then by (22) we obtain

$$
\begin{gathered}
\int_{\prod_{i=1}^{N}\left(a_{i}, b_{i}\right)} u_{1}(x) \prod_{j=1}^{m} \Phi_{j}\left(\frac{\left|I_{a+}^{\alpha}\left(f_{j}\right)(x)\right| \prod_{i=1}^{N} \Gamma\left(\alpha_{i}+1\right)}{\prod_{i=1}^{N}\left(x_{i}-a_{i}\right)^{\alpha_{i}}}\right) d x \\
\leq \prod_{j=1}^{m}\left(\int_{\prod_{i=1}^{N}\left(a_{i}, b_{i}\right)} w_{1}(y) \Phi_{j}\left(\left|f_{j}(y)\right|\right)^{p_{j}} d y\right)^{\frac{1}{p_{j}}}
\end{gathered}
$$

under the assumptions:

(i) $f_{j}, \Phi_{j}\left(\left|f_{j}\right|\right)^{p_{j}}$ are both $\frac{1}{\prod_{i=1}^{N} \Gamma\left(\alpha_{i}\right)} \chi_{\prod_{i=1}^{N}\left(a_{i}, x_{i}\right]}(y) \prod_{i=1}^{N}\left(x_{i}-y_{i}\right)^{\alpha_{i}-1} d y$ -integrable, a.e. in $x \in \prod_{i=1}^{N}\left(a_{i}, b_{i}\right)$, for all $j=1, \ldots, m$,

(ii) $w_{1} \Phi_{j}\left(\left|f_{j}\right|\right)^{p_{j}}$ is Lebesgue integrable, $j=1, \ldots, m$.

Similarly, by (22), we obtain

$$
\int_{\prod_{i=1}^{N}\left(a_{i}, b_{i}\right)} u_{2}(x) \prod_{j=1}^{m} \Phi_{j}\left(\frac{\left|I_{b-}^{\alpha}\left(f_{j}\right)(x)\right| \prod_{i=1}^{N} \Gamma\left(\alpha_{i}+1\right)}{\prod_{i=1}^{N}\left(b_{i}-x_{i}\right)^{\alpha_{i}}}\right) d x
$$




$$
\leq \prod_{j=1}^{m}\left(\int_{\prod_{i=1}^{N}\left(a_{i}, b_{i}\right)} w_{2}(y) \Phi_{j}\left(\left|f_{j}(y)\right|\right)^{p_{j}} d y\right)^{\frac{1}{p_{j}}},
$$

under the assumptions:

(i) $f_{j}, \Phi_{j}\left(\left|f_{j}\right|\right)^{p_{j}}$ are both $\frac{1}{\prod_{i=1}^{N} \Gamma\left(\alpha_{i}\right)} \chi_{\prod_{i=1}^{N}\left[x_{i}, b_{i}\right)}(y) \prod_{i=1}^{N}\left(y_{i}-x_{i}\right)^{\alpha_{i}-1} d y$ -integrable, a.e. in $x \in \prod_{i=1}^{N}\left(a_{i}, b_{i}\right)$, for all $j=1, \ldots, m$,

(ii) $w_{2} \Phi_{j}\left(\left|f_{j}\right|\right)^{p_{j}}$ is Lebesgue integrable, $j=1, \ldots, m$.

Using (72) and (73) we rewrite (76), as follows

$$
\begin{gathered}
\int_{\prod_{i=1}^{N}\left(a_{i}, b_{i}\right)}\left(\prod_{i=1}^{N}\left(x_{i}-a_{i}\right)^{\alpha_{i}}\right) \prod_{j=1}^{m} \Phi_{j}\left(\frac{\left|I_{a+}^{\alpha}\left(f_{j}\right)(x)\right| \prod_{i=1}^{N} \Gamma\left(\alpha_{i}+1\right)}{\prod_{i=1}^{N}\left(x_{i}-a_{i}\right)^{\alpha_{i}}}\right) d x \\
\leq \prod_{j=1}^{m}\left(\int_{\prod_{i=1}^{N}\left(a_{i}, b_{i}\right)}\left(\prod_{i=1}^{N}\left(b_{i}-y_{i}\right)^{\alpha_{i}}\right) \Phi_{j}\left(\left|f_{j}(y)\right|\right)^{p_{j}} d y\right)^{\frac{1}{p_{j}}} \leq \\
\left(\prod_{i=1}^{N}\left(b_{i}-a_{i}\right)^{\alpha_{i}}\right) \prod_{j=1}^{m}\left(\int_{\prod_{i=1}^{N}\left(a_{i}, b_{i}\right)} \Phi_{j}\left(\left|f_{j}(y)\right|\right)^{p_{j}} d y\right)^{\frac{1}{p_{j}}},
\end{gathered}
$$

under the assumptions:

(i) following (76) and

(ii)* $\Phi_{j}\left(\left|f_{j}\right|\right)^{p_{j}}$ is Lebesgue integrable, $j=1, \ldots, m$.

Similarly, using (74) and (75) we rewrite (77),

$$
\begin{gathered}
\int_{\prod_{i=1}^{N}\left(a_{i}, b_{i}\right)}\left(\prod_{i=1}^{N}\left(b_{i}-x_{i}\right)^{\alpha_{i}}\right) \prod_{j=1}^{m} \Phi_{j}\left(\frac{\left|I_{b-}^{\alpha}\left(f_{j}\right)(x)\right| \prod_{i=1}^{N} \Gamma\left(\alpha_{i}+1\right)}{\prod_{i=1}^{N}\left(b_{i}-x_{i}\right)^{\alpha_{i}}}\right) d x \\
\leq \prod_{j=1}^{m}\left(\int_{\prod_{i=1}^{N}\left(a_{i}, b_{i}\right)}\left(\prod_{i=1}^{N}\left(y_{i}-a_{i}\right)^{\alpha_{i}}\right) \Phi_{j}\left(\left|f_{j}(y)\right|\right)^{p_{j}} d y\right)^{\frac{1}{p_{j}}} \\
\leq\left(\prod_{i=1}^{N}\left(b_{i}-a_{i}\right)^{\alpha_{i}}\right) \prod_{j=1}^{m}\left(\int_{\prod_{i=1}^{N}\left(a_{i}, b_{i}\right)} \Phi_{j}\left(\left|f_{j}(y)\right|\right)^{p_{j}} d y\right)^{\frac{1}{p_{j}}}
\end{gathered}
$$

under the assumptions:

(i) following (77), and 
(ii) $^{*} \Phi_{j}\left(\left|f_{j}\right|\right)^{p_{j}}$ is Lebesgue integrable, $j=1, \ldots, m$.

Let now $\beta_{j} \geq 1, j=1, \ldots, m$.

Then, by (78), we obtain

$$
\begin{gathered}
\int_{\prod_{i=1}^{N}\left(a_{i}, b_{i}\right)}\left(\prod_{i=1}^{N}\left(x_{i}-a_{i}\right)^{\alpha_{i}}\right)^{\left(1-\sum_{j=1}^{m} \beta_{j}\right)}\left(\prod_{j=1}^{m}\left|I_{a+}^{\alpha}\left(f_{j}\right)(x)\right|^{\beta_{j}}\right) d x \\
\leq\left(\frac{1}{\left(\prod_{i=1}^{N} \Gamma\left(\alpha_{i}+1\right)\right)^{\sum_{j=1}^{m} \beta_{j}}}\right) \\
\leq\left(\frac { \prod _ { j = 1 } ^ { m } ( \int _ { \prod _ { i = 1 } ^ { N } ( a _ { i } , b _ { i } ) } ( \prod _ { i = 1 } ^ { N } ( b _ { i } - y _ { i } ) ^ { \alpha _ { i } } ) | f _ { j } ( y ) | ^ { \beta _ { j } p _ { j } } d y ) ^ { \frac { 1 } { p _ { j } } } } { ( \prod _ { i = 1 } ^ { N } ( b _ { i } - a _ { i } ) ^ { \alpha _ { i } } \prod _ { i = 1 } ^ { m } \Gamma ( \alpha _ { i } + 1 ) ) ^ { \sum _ { j = 1 } ^ { m } \beta _ { j } } } \prod _ { j = 1 } ^ { m } \left(\int_{\prod_{i=1}^{N}\left(a_{i}, b_{i}\right)}^{\left.\left|f_{j}(y)\right|^{\beta_{j} p_{j}} d y\right)^{\frac{1}{p_{j}}}} .\right.\right.
\end{gathered}
$$

But it holds

$$
\begin{aligned}
& \int_{\prod_{i=1}^{N}\left(a_{i}, b_{i}\right)}\left(\prod_{i=1}^{N}\left(x_{i}-a_{i}\right)^{\alpha_{i}}\right)^{\left(1-\sum_{j=1}^{m} \beta_{j}\right)}\left(\prod_{j=1}^{m}\left|I_{a+}^{\alpha}\left(f_{j}\right)(x)\right|^{\beta_{j}}\right) d x \\
\geq & \left(\prod_{i=1}^{N}\left(b_{i}-a_{i}\right)^{\alpha_{i}}\right)^{\left(1-\sum_{j=1}^{m} \beta_{j}\right)}\left(\int_{\prod_{i=1}^{N}\left(a_{i}, b_{i}\right)} \prod_{j=1}^{m}\left|I_{a+}^{\alpha}\left(f_{j}\right)(x)\right|^{\beta_{j}} d x\right) .
\end{aligned}
$$

So by (80) and (81) we derive

$$
\begin{gathered}
\int_{\prod_{i=1}^{N}\left(a_{i}, b_{i}\right)} \prod_{j=1}^{m}\left|I_{a+}^{\alpha}\left(f_{j}\right)(x)\right|^{\beta_{j}} d x \\
\leq\left(\prod_{i=1}^{N} \frac{\left(b_{i}-a_{i}\right)^{\alpha_{i}}}{\Gamma\left(\alpha_{i}+1\right)}\right)^{\sum_{j=1}^{m} \beta_{j}} \prod_{j=1}^{m}\left(\int_{\prod_{i=1}^{N}\left(a_{i}, b_{i}\right)}\left|f_{j}(y)\right|^{\beta_{j} p_{j}} d y\right)^{\frac{1}{p_{j}}},
\end{gathered}
$$

under the assumptions:

(i) $\left|f_{j}\right|^{p_{j} \beta_{j}}$ is $\frac{1}{\prod_{i=1}^{N} \Gamma\left(\alpha_{i}\right)} \chi_{\prod_{i=1}^{N}\left(a_{i}, x_{i}\right]}(y) \prod_{i=1}^{N}\left(x_{i}-y_{i}\right)^{\alpha_{i}-1} d y$-integrable, a.e. in $x \in \prod_{i=1}^{N}\left(a_{i}, b_{i}\right)$, for all $j=1, \ldots, m$, 
(ii) $\left|f_{j}\right|^{p_{j} \beta_{j}}$ is Lebesgue integrable, $j=1, \ldots, m$.

We also have, by (78), that

$$
\begin{gathered}
\int_{\prod_{i=1}^{N}\left(a_{i}, b_{i}\right)}\left(\prod_{i=1}^{N}\left(x_{i}-a_{i}\right)^{\alpha_{i}}\right) e^{\left(\sum_{j=1}^{m}\left|I_{a+}^{\alpha}\left(f_{j}\right)(x)\right|\right)\left(\prod_{i=1}^{N} \frac{\Gamma\left(\alpha_{i}+1\right)}{\left(x_{i}-a_{i}\right)^{\alpha_{i}}}\right)} d x \\
\leq \prod_{j=1}^{m}\left(\int_{\prod_{i=1}^{N}\left(a_{i}, b_{i}\right)}\left(\prod_{i=1}^{N}\left(b_{i}-y_{i}\right)^{\alpha_{i}}\right) e^{p_{j}\left|f_{j}(y)\right|} d y\right)^{\frac{1}{p_{j}}} \\
\leq\left(\prod_{i=1}^{N}\left(b_{i}-a_{i}\right)^{\alpha_{i}}\right) \prod_{j=1}^{m}\left(\int_{\prod_{i=1}^{N}\left(a_{i}, b_{i}\right)} e^{p_{j}\left|f_{j}(y)\right|} d y\right)^{\frac{1}{p_{j}}},
\end{gathered}
$$

under the assumptions:

(i) $f_{j}, e^{p_{j}\left|f_{j}\right|}$ are both $\frac{1}{\prod_{i=1}^{N} \Gamma\left(\alpha_{i}\right)} \chi_{\prod_{i=1}^{N}\left(a_{i}, x_{i}\right]}(y) \prod_{i=1}^{N}\left(x_{i}-y_{i}\right)^{\alpha_{i}-1} d y-$ integrable, a.e. in $x \in \prod_{i=1}^{N}\left(a_{i}, b_{i}\right)$, for all $j=1, \ldots, m$,

(ii) $e^{p_{j}\left|f_{j}\right|}$ is Lebesgue integrable, $j=1, \ldots, m$.

From (79) we get

$$
\begin{gathered}
\int_{\prod_{i=1}^{N}\left(a_{i}, b_{i}\right)}\left(\prod_{i=1}^{N}\left(b_{i}-x_{i}\right)^{\alpha_{i}}\right)^{\left(1-\sum_{j=1}^{m} \beta_{j}\right)}\left(\prod_{j=1}^{m}\left|I_{b-}^{\alpha}\left(f_{j}\right)(x)\right|^{\beta_{j}}\right) d x \\
\leq\left(\frac{1}{\left(\prod_{i=1}^{N} \Gamma\left(\alpha_{i}+1\right)\right)^{\sum_{j=1}^{m} \beta_{j}}}\right) \\
\cdot \prod_{j=1}^{m}\left(\int_{\prod_{i=1}^{N}\left(a_{i}, b_{i}\right)}^{\left.\left(\prod_{i=1}^{N}\left(y_{i}-a_{i}\right)^{\alpha_{i}}\right)\left|f_{j}(y)\right|^{\beta_{j} p_{j}} d y\right)^{\frac{1}{p_{j}}}}\right. \\
\leq\left(\frac{\prod_{i=1}^{N}\left(b_{i}-a_{i}\right)^{\alpha_{i}}}{\left(\prod_{i=1}^{N} \Gamma\left(\alpha_{i}+1\right)\right)^{\sum_{j=1}^{m} \beta_{j}}}\right) \prod_{j=1}^{m}\left(\int_{\prod_{i=1}^{N}\left(a_{i}, b_{i}\right)}\left|f_{j}(y)\right|^{\beta_{j} p_{j}} d y\right)^{\frac{1}{p_{j}}}
\end{gathered}
$$


But it holds

$$
\begin{gathered}
\int_{\prod_{i=1}^{N}\left(a_{i}, b_{i}\right)}\left(\prod_{i=1}^{N}\left(b_{i}-x_{i}\right)^{\alpha_{i}}\right)^{\left(1-\sum_{j=1}^{m} \beta_{j}\right)}\left(\prod_{j=1}^{m}\left|I_{b-}^{\alpha}\left(f_{j}\right)(x)\right|^{\beta_{j}}\right) d x \\
\geq\left(\prod_{i=1}^{N}\left(b_{i}-a_{i}\right)^{\alpha_{i}}\right)^{\left(1-\sum_{j=1}^{m} \beta_{j}\right)}\left(\int_{\prod_{i=1}^{N}\left(a_{i}, b_{i}\right)}\left(\prod_{j=1}^{m}\left|I_{b-}^{\alpha}\left(f_{j}\right)(x)\right|^{\beta_{j}}\right) d x\right) .
\end{gathered}
$$

So by (84) and (85) we obtain

$$
\begin{gathered}
\int_{\prod_{i=1}^{N}\left(a_{i}, b_{i}\right)}\left(\prod_{j=1}^{m}\left|I_{b-}^{\alpha}\left(f_{j}\right)(x)\right|^{\beta_{j}}\right) d x \\
\leq\left(\prod_{i=1}^{N} \frac{\left(b_{i}-a_{i}\right)^{\alpha_{i}}}{\Gamma\left(\alpha_{i}+1\right)}\right)^{\sum_{j=1}^{m} \beta_{j}} \prod_{j=1}^{m}\left(\int_{\prod_{i=1}^{N}\left(a_{i}, b_{i}\right)}\left|f_{j}(y)\right|^{\beta_{j} p_{j}} d y\right)^{\frac{1}{p_{j}}},
\end{gathered}
$$

under the assumptions:

(i) $\left|f_{j}\right|^{p_{j} \beta_{j}}$ is $\frac{1}{\prod_{i=1}^{N} \Gamma\left(\alpha_{i}\right)} \chi_{\prod_{i=1}^{N}\left[x_{i}, b_{i}\right)}(y) \prod_{i=1}^{N}\left(y_{i}-x_{i}\right)^{\alpha_{i}-1} d y$-integrable, a.e. in $x \in \prod_{i=1}^{N}\left(a_{i}, b_{i}\right)$, for all $j=1, \ldots, m$,

(ii) $\left|f_{j}\right|^{p_{j} \beta_{j}}$ is Lebesgue integrable, $j=1, \ldots, m$.

We also have, by (79), that

$$
\begin{gathered}
\int_{\prod_{i=1}^{N}\left(a_{i}, b_{i}\right)}\left(\prod_{i=1}^{N}\left(b_{i}-x_{i}\right)^{\alpha_{i}}\right) e^{\left(\sum_{j=1}^{m}\left|I_{b-}^{\alpha}\left(f_{j}\right)(x)\right|\right)\left(\prod_{i=1}^{N} \frac{\Gamma\left(\alpha_{i}+1\right)}{\left(b_{i}-x_{i}\right)^{\alpha_{i}}}\right)} d x \\
\leq \prod_{j=1}^{m}\left(\int_{\prod_{i=1}^{N}\left(a_{i}, b_{i}\right)}\left(\prod_{i=1}^{N}\left(y_{i}-a_{i}\right)^{\alpha_{i}}\right) e^{p_{j}\left|f_{j}(y)\right|} d y\right)^{\frac{1}{p_{j}}} \\
\leq\left(\prod_{i=1}^{N}\left(b_{i}-a_{i}\right)^{\alpha_{i}}\right) \prod_{j=1}^{m}\left(\int_{\prod_{i=1}^{N}\left(a_{i}, b_{i}\right)} e^{p_{j}\left|f_{j}(y)\right|} d y\right)^{\frac{1}{p_{j}}},
\end{gathered}
$$

under the assumptions:

(i) $f_{j}, e^{p_{j}\left|f_{j}\right|}$ are both $\frac{1}{\prod_{i=1}^{N} \Gamma\left(\alpha_{i}\right)} \chi_{\prod_{i=1}^{N}\left[x_{i}, b_{i}\right)}(y) \prod_{i=1}^{N}\left(y_{i}-x_{i}\right)^{\alpha_{i}-1} d y-$ integrable, a.e. in $x \in \prod_{i=1}^{N}\left(a_{i}, b_{i}\right)$, for all $j=1, \ldots, m$,

(ii) $e^{p_{j}\left|f_{j}\right|}$ is Lebesgue integrable, $j=1, \ldots, m$. 
Background 20. In order to apply Theorem 1 to the case of a spherical shell we need:

Let $N \geq 2, S^{N-1}:=\left\{x \in \mathbb{R}^{N}:|x|=1\right\}$ the unit sphere on $\mathbb{R}^{N}$, where $|\cdot|$ stands for the Euclidean norm in $\mathbb{R}^{N}$. Also denote the ball $B(0, R):=\{x \in$ $\left.\mathbb{R}^{N}:|x|<R\right\} \subseteq \mathbb{R}^{N}, R>0$, and the spherical shell

$$
A:=B\left(0, R_{2}\right)-\overline{B\left(0, R_{1}\right)}, \quad 0<R_{1}<R_{2} .
$$

For the following see [15, pp. 149-150], and [17, pp. 87-88].

For $x \in \mathbb{R}^{N}-\{0\}$ we can write uniquely $x=r \omega$, where $r=|x|>0$, and $\omega=\frac{x}{r} \in S^{N-1},|\omega|=1$.

Clearly here

$$
\mathbb{R}^{N}-\{0\}=(0, \infty) \times S^{N-1},
$$

and

$$
\bar{A}=\left[R_{1}, R_{2}\right] \times S^{N-1} .
$$

We will be using

Theorem 21. [1, p. 322] Let $f: A \rightarrow \mathbb{R}$ be a Lebesgue integrable function. Then

$$
\int_{A} f(x) d x=\int_{S^{N-1}}\left(\int_{R_{1}}^{R_{2}} f(r \omega) r^{N-1} d r\right) d \omega .
$$

So we are able to write an integral on the shell in polar form using the polar coordinates $(r, \omega)$.

We need

Definition 22. [1, p. 458] Let $\nu>0, n:=[\nu], \alpha:=\nu-n, f \in C^{n}(\bar{A})$, and $A$ is a spherical shell. Assume that there exists function $\frac{\partial_{R_{1}}^{\nu} f(x)}{\partial r^{\nu}} \in C(\bar{A})$, given by

$$
\frac{\partial_{R_{1}}^{\nu} f(x)}{\partial r^{\nu}}:=\frac{1}{\Gamma(1-\alpha)} \frac{\partial}{\partial r}\left(\int_{R_{1}}^{r}(r-t)^{-\alpha} \frac{\partial^{n} f(t \omega)}{\partial r^{n}} d t\right),
$$

where $x \in \bar{A}$; that is $x=r \omega, r \in\left[R_{1}, R_{2}\right], \omega \in S^{N-1}$.

We call $\frac{\partial_{R_{1}}^{\nu}}{\partial r^{\nu}}$ the left radial Canavati-type fractional derivative of $f$ of order $\nu$. If $\nu=0$, then set $\frac{\partial_{R_{1}}^{\nu} f(x)}{\partial r^{\nu}}:=f(x)$.

Based on [1, p. 288], and [5] we have

Lemma 23. Let $\gamma \geq 0, m:=[\gamma], \nu>0, n:=[\nu]$, with $0 \leq \gamma<\nu$. Let $f \in C^{n}(\bar{A})$ and there exists $\frac{\partial_{R_{1}}^{\nu} f(x)}{\partial r^{\nu}} \in C(\bar{A}), x \in \bar{A}, A$ a sperical shell. 
Further assume that $\frac{\partial^{j} f\left(R_{1} \omega\right)}{\partial r^{j}}=0, j=m, m+1, \ldots, n-1, \forall \omega \in S^{N-1}$. Then there exists $\frac{\partial_{R_{1}}^{\gamma} f(x)}{\partial r^{\gamma}} \in C(\bar{A})$ such that

$$
\frac{\partial_{R_{1}}^{\gamma} f(x)}{\partial r^{\gamma}}=\frac{\partial_{R_{1}}^{\gamma} f(r \omega)}{\partial r^{\gamma}}=\frac{1}{\Gamma(\nu-\gamma)} \int_{R_{1}}^{r}(r-t)^{\nu-\gamma-1} \frac{\partial_{R_{1}}^{\nu} f(t \omega)}{\partial r^{\nu}} d t
$$

$\forall \omega \in S^{N-1} ;$ all $R_{1} \leq r \leq R_{2}$, indeed $f(r \omega) \in C_{R_{1}}^{\gamma}\left(\left[R_{1}, R_{2}\right]\right), \forall \omega \in S^{N-1}$.

We make

Remark 24. In the settings and assumptions of Theorem 1 and Lemma 23 we have

$$
k(r, t)=\frac{1}{\Gamma(\nu-\gamma)} \chi_{\left[R_{1}, r\right]}(t)(r-t)^{\nu-\gamma-1},
$$

and

$$
K(r)=\frac{\left(r-R_{1}\right)^{\nu-\gamma}}{\Gamma(\nu-\gamma+1)}
$$

$r, t \in\left[R_{1}, R_{2}\right]$.

Furthermore we get

$$
\frac{k(r, t)}{K(r)}=(\nu-\gamma) \chi_{\left[R_{1}, r\right]}(t) \frac{(r-t)^{\nu-\gamma-1}}{\left(r-R_{1}\right)^{\nu-\gamma}},
$$

and by choosing

$$
u(r):=\left(r-R_{1}\right)^{\nu-\gamma}, \quad r \in\left[R_{1}, R_{2}\right],
$$

we find

$$
U(t)=(\nu-\gamma) \int_{t}^{R_{2}}(r-t)^{\nu-\gamma-1} d r=\left(R_{2}-t\right)^{\nu-\gamma},
$$

$t \in\left[R_{1}, R_{2}\right]$.

Then by (8) for $p \geq 1$ we find

$$
\begin{gathered}
\int_{R_{1}}^{R_{2}}\left(r-R_{1}\right)^{\nu-\gamma}\left|\frac{\partial_{R_{1}}^{\gamma} f(r \omega)}{\partial r^{\gamma}}\right|^{p} \frac{(\Gamma(\nu-\gamma+1))^{p}}{\left(r-R_{1}\right)^{(\nu-\gamma) p}} d r \\
\leq \int_{R_{1}}^{R_{2}}\left(R_{2}-r\right)^{\nu-\gamma}\left|\frac{\partial_{R_{1}}^{\nu} f(r \omega)}{\partial r^{\nu}}\right|^{p} d r
\end{gathered}
$$

and

$$
\begin{gathered}
\int_{R_{1}}^{R_{2}}\left(r-R_{1}\right)^{(\nu-\gamma)(1-p)}\left|\frac{\partial_{R_{1}}^{\gamma} f(r \omega)}{\partial r^{\gamma}}\right|^{p} d r \\
\leq \frac{1}{(\Gamma(\nu-\gamma+1))^{p}} \int_{R_{1}}^{R_{2}}\left(R_{2}-r\right)^{\nu-\gamma}\left|\frac{\partial_{R_{1}}^{\nu} f(r \omega)}{\partial r^{\nu}}\right|^{p} d r
\end{gathered}
$$




$$
\leq \frac{\left(R_{2}-R_{1}\right)^{\nu-\gamma}}{(\Gamma(\nu-\gamma+1))^{p}} \int_{R_{1}}^{R_{2}}\left|\frac{\partial_{R_{1}}^{\nu} f(r \omega)}{\partial r^{\nu}}\right|^{p} d r
$$

But it holds

$$
\begin{gathered}
\int_{R_{1}}^{R_{2}}\left(r-R_{1}\right)^{(\nu-\gamma)(1-p)}\left|\frac{\partial_{R_{1}}^{\gamma} f(r \omega)}{\partial r^{\gamma}}\right|^{p} d r \\
\geq\left(R_{2}-R_{1}\right)^{(\nu-\gamma)(1-p)} \int_{R_{1}}^{R_{2}}\left|\frac{\partial_{R_{1}}^{\gamma} f(r \omega)}{\partial r^{\gamma}}\right|^{p} d r .
\end{gathered}
$$

Consequently we derive

$$
\int_{R_{1}}^{R_{2}}\left|\frac{\partial_{R_{1}}^{\gamma} f(r \omega)}{\partial r^{\gamma}}\right|^{p} d r \leq\left(\frac{\left(R_{2}-R_{1}\right)^{(\nu-\gamma)}}{\Gamma(\nu-\gamma+1)}\right)^{p} \int_{R_{1}}^{R_{2}}\left|\frac{\partial_{R_{1}}^{\nu} f(r \omega)}{\partial r^{\nu}}\right|^{p} d r,
$$

$\forall \omega \in S^{N-1}$.

Here we have $R_{1} \leq r \leq R_{2}$, and $R_{1}^{N-1} \leq r^{N-1} \leq R_{2}^{N-1}$, and $R_{2}^{1-N} \leq$ $r^{1-N} \leq R_{1}^{1-N}$.

From (102) we have

$$
\begin{gathered}
R_{2}^{1-N} \int_{R_{1}}^{R_{2}} r^{N-1}\left|\frac{\partial_{R_{1}}^{\gamma} f(r \omega)}{\partial r^{\gamma}}\right|^{p} d r \leq \int_{R_{1}}^{R_{2}} r^{1-N} r^{N-1}\left|\frac{\partial_{R_{1}}^{\gamma} f(r \omega)}{\partial r^{\gamma}}\right|^{p} d r \\
\leq\left(\frac{\left(R_{2}-R_{1}\right)^{(\nu-\gamma)}}{\Gamma(\nu-\gamma+1)}\right)^{p} \int_{R_{1}}^{R_{2}} r^{1-N} r^{N-1}\left|\frac{\partial_{R_{1}}^{\nu} f(r \omega)}{\partial r^{\nu}}\right|^{p} d r \\
\leq R_{1}^{1-N}\left(\frac{\left(R_{2}-R_{1}\right)^{(\nu-\gamma)}}{\Gamma(\nu-\gamma+1)}\right)^{p} \int_{R_{1}}^{R_{2}} r^{N-1}\left|\frac{\partial_{R_{1}}^{\nu} f(r \omega)}{\partial r^{\nu}}\right|^{p} d r .
\end{gathered}
$$

So we get

$$
\begin{gathered}
\int_{R_{1}}^{R_{2}} r^{N-1}\left|\frac{\partial_{R_{1}}^{\gamma} f(r \omega)}{\partial r^{\gamma}}\right|^{p} d r \\
\leq\left(\frac{R_{2}}{R_{1}}\right)^{N-1}\left(\frac{\left(R_{2}-R_{1}\right)^{(\nu-\gamma)}}{\Gamma(\nu-\gamma+1)}\right)^{p} \int_{R_{1}}^{R_{2}} r^{N-1}\left|\frac{\partial_{R_{1}}^{\nu} f(r \omega)}{\partial r^{\nu}}\right|^{p} d r
\end{gathered}
$$

$\forall \omega \in S^{N-1}$.

Hence

$$
\begin{gathered}
\int_{S^{N-1}}\left(\int_{R_{1}}^{R_{2}} r^{N-1}\left|\frac{\partial_{R_{1}}^{\gamma} f(r \omega)}{\partial r^{\gamma}}\right|^{p} d r\right) d \omega \\
\leq\left(\frac{R_{2}}{R_{1}}\right)^{N-1}\left(\frac{\left(R_{2}-R_{1}\right)^{(\nu-\gamma)}}{\Gamma(\nu-\gamma+1)}\right)^{p} \int_{S^{N-1}}\left(\int_{R_{1}}^{R_{2}} r^{N-1}\left|\frac{\partial_{R_{1}}^{\nu} f(r \omega)}{\partial r^{\nu}}\right|^{p} d r\right) d \omega .
\end{gathered}
$$


By Theorem 21, equality (91), we obtain

$$
\int_{A}\left|\frac{\partial_{R_{1}}^{\gamma} f(x)}{\partial r^{\gamma}}\right|^{p} d x \leq\left(\frac{R_{2}}{R_{1}}\right)^{N-1}\left(\frac{\left(R_{2}-R_{1}\right)^{(\nu-\gamma)}}{\Gamma(\nu-\gamma+1)}\right)^{p} \int_{A}\left|\frac{\partial_{R_{1}}^{\nu} f(x)}{\partial r^{\nu}}\right|^{p} d x .
$$

We have proved the following fractional Poincaré type inequalities on the shell.

Theorem 25. Here all as in Lemma 23, $p \geq 1$.

It holds

1)

$$
\left\|\frac{\partial_{R_{1}}^{\gamma} f}{\partial r^{\gamma}}\right\|_{p, A} \leq\left(\frac{R_{2}}{R_{1}}\right)^{\left(\frac{N-1}{p}\right)}\left(\frac{\left(R_{2}-R_{1}\right)^{(\nu-\gamma)}}{\Gamma(\nu-\gamma+1)}\right)\left\|\frac{\partial_{R_{1}}^{\nu} f}{\partial r^{\nu}}\right\|_{p, A}
$$

2) When $\gamma=0$, we have

$$
\|f\|_{p, A} \leq\left(\frac{R_{2}}{R_{1}}\right)^{\left(\frac{N-1}{p}\right)}\left(\frac{\left(R_{2}-R_{1}\right)^{\nu}}{\Gamma(\nu+1)}\right)\left\|\frac{\partial_{R_{1}}^{\nu} f}{\partial r^{\nu}}\right\|_{p, A} .
$$

See the related, and proof, results in [1, pp. 458-459] with different constants and proof in the corresponding inequalities.

Similar results can be produced for the right radial Canavati type fractional derivative. We choose to omit it.

We make

Remark 26. (from [1], p. 460) Here we denote $\lambda_{\mathbb{R}^{N}}(x) \equiv d x$ the Lebesgue measure on $\mathbb{R}^{N}, N \geq 2$, and by $\lambda_{S^{N-1}}(\omega)=d \omega$ the surface measure on $S^{N-1}$, where $\mathcal{B}_{X}$ stands for the Borel class on space $X$. Define the measure $R_{N}$ on $\left((0, \infty), \mathcal{B}_{(0, \infty)}\right)$ by

$$
R_{N}(B)=\int_{B} r^{N-1} d r, \text { any } B \in \mathcal{B}_{(0, \infty)} .
$$

Now let $F \in L_{1}(A)=L_{1}\left(\left[R_{1}, R_{2}\right] \times S^{N-1}\right)$.

Call

$$
K(F):=\left\{\omega \in S^{N-1}: F(\cdot \omega) \notin L_{1}\left(\left[R_{1}, R_{2}\right], \mathcal{B}_{\left[R_{1}, R_{2}\right]}, R_{N}\right)\right\} .
$$

We get, by Fubini's theorem and [17, pp. 87-88] that

$$
\lambda_{S^{N-1}}(K(F))=0 .
$$

Of course

$$
\theta(F):=\left[R_{1}, R_{2}\right] \times K(F) \subset A,
$$

and

$$
\lambda_{\mathbb{R}^{N}}(\theta(F))=0 .
$$


Above $\lambda_{S^{N-1}}$ is defined as follows: let $A \subset S^{N-1}$ be a Borel set, and let

$$
\widetilde{A}:=\{r u: 0<r<1, u \in A\} \subset \mathbb{R}^{N} ;
$$

we define

$$
\lambda_{S^{N-1}}(A):=N \lambda_{\mathbb{R}^{N}}(\widetilde{A})
$$

We have that

$$
\lambda_{S^{N-1}}\left(S^{N-1}\right)=\frac{2 \pi^{\frac{N}{2}}}{\Gamma\left(\frac{N}{2}\right)},
$$

the surface area of $S^{N-1}$.

See also [15, pp. 149-150], [17, pp. 87-88] and [1, p. 320].

Following $[1$, p. 466] we define the left Riemann-Liouville radial fractional derivative next.

Definition 27. Let $\beta>0, m:=[\beta]+1, F \in L_{1}(A)$, and $A$ is the spherical shell. We define

$$
\frac{\bar{\partial}_{R_{1}}^{\beta} F(x)}{\partial r^{\beta}}:=\left\{\begin{array}{l}
\frac{1}{\Gamma(m-\beta)}\left(\frac{\partial}{\partial r}\right)^{m} \int_{R_{1}}^{r}(r-t)^{m-\beta-1} F(t \omega) d t, \\
\text { for } \omega \in S^{N-1}-K(F), \quad \text { for } \omega \in K(F),
\end{array}\right.
$$

where $x=r \omega \in A, r \in\left[R_{1}, R_{2}\right], \omega \in S^{N-1} ; K(F)$ as in (109).

If $\beta=0$, define

$$
\frac{\bar{\partial}_{R_{1}}^{\beta} F(x)}{\partial r^{\beta}}:=F(x)
$$

We need the following important representation result for left RiemannLiouville radial fractional derivatives, by [1, p. 466].

Theorem 28. Let $\nu \geq \gamma+1, \gamma \geq 0, n:=[\nu], m:=[\gamma], F: \bar{A} \rightarrow \mathbb{R}$ with $F \in L_{1}(A)$. Assume that $F(\cdot \omega) \in A C^{n}\left(\left[R_{1}, R_{2}\right]\right), \forall \omega \in S^{N-1}$, and that $\frac{\bar{\partial}_{R_{1}}^{\nu} F(\cdot \omega)}{\partial r^{\nu}}$ is measurable on $\left[R_{1}, R_{2}\right], \forall \omega \in S^{N-1}$. Also assume $\exists$ $\frac{\bar{\partial}_{R_{1}}^{\nu} F(r \omega)}{\partial r^{\nu}} \in \mathbb{R}, \forall r \in\left[R_{1}, R_{2}\right]$ and $\forall \omega \in S^{N-1}$, and $\frac{\bar{\partial}_{R_{1}}^{\nu} F(x)}{\partial r^{\nu}}$ is measurable on $\bar{A}$. Suppose $\exists M_{1}>0$ :

$$
\left|\frac{\bar{\partial}_{R_{1}}^{\nu} F(r \omega)}{\partial r^{\nu}}\right| \leq M_{1}, \quad \forall(r, \omega) \in\left[R_{1}, R_{2}\right] \times S^{N-1} .
$$

We suppose that $\frac{\bar{\partial}^{j} F\left(R_{1} \omega\right)}{\partial r^{j}}=0, j=m, m+1, \ldots, n-1 ; \forall \omega \in S^{N-1}$.

Then

$$
\frac{\bar{\partial}_{R_{1}}^{\gamma} F(x)}{\partial r^{\gamma}}=\bar{D}_{R_{1}}^{\gamma} F(r \omega)=\frac{1}{\Gamma(\nu-\gamma)} \int_{R_{1}}^{r}(r-t)^{\nu-\gamma-1}\left(\bar{D}_{R_{1}}^{\nu} F\right)(t \omega) d t,
$$


valid $\forall x \in \bar{A}$; that is, true $\forall r \in\left[R_{1}, R_{2}\right]$ and $\forall \omega \in S^{N-1} ; \gamma>0$.

Here

$$
\bar{D}_{R_{1}}^{\gamma} F(\cdot \omega) \in A C\left(\left[R_{1}, R_{2}\right]\right),
$$

$\forall \omega \in S^{N-1} ; \gamma>0$.

Furthermore

In particular, it holds

$$
\frac{\bar{\partial}_{R_{1}}^{\gamma} F(x)}{\partial r^{\gamma}} \in L_{\infty}(A), \gamma>0
$$

$$
F(x)=F(r \omega)=\frac{1}{\Gamma(\nu)} \int_{R_{1}}^{r}(r-t)^{\nu-1}\left(\bar{D}_{R_{1}}^{\nu} F\right)(t \omega) d t,
$$

true $\forall x \in \bar{A}$; that is, true $\forall r \in\left[R_{1}, R_{2}\right]$ and $\forall \omega \in S^{N-1}$, and

$$
F(\cdot \omega) \in A C\left(\left[R_{1}, R_{2}\right]\right), \quad \forall \omega \in S^{N-1} .
$$

We give also the following fractional Poincaré type inequalities on the spherical shell.

Theorem 29. Here all as in Theorem $28, p \geq 1$. Then

1)

$$
\left\|\frac{\bar{\partial}_{R_{1}}^{\gamma} F}{\partial r^{\gamma}}\right\|_{p, A} \leq\left(\frac{R_{2}}{R_{1}}\right)^{\left(\frac{N-1}{p}\right)}\left(\frac{\left(R_{2}-R_{1}\right)^{(\nu-\gamma)}}{\Gamma(\nu-\gamma+1)}\right)\left\|\frac{\bar{\partial}_{R_{1}}^{\nu} F}{\partial r^{\nu}}\right\|_{p, A},
$$

2) When $\gamma=0$, we have

$$
\|F\|_{p, A} \leq\left(\frac{R_{2}}{R_{1}}\right)^{\left(\frac{N-1}{p}\right)}\left(\frac{\left(R_{2}-R_{1}\right)^{\nu}}{\Gamma(\nu+1)}\right)\left\|\frac{\bar{\partial}_{R_{1}}^{\nu} F}{\partial r^{\nu}}\right\|_{p, A} .
$$

Proof. As in Theorem 25, based on Theorem 28.

See also similar results in [1, p. 468].

We also need (see [1], p. 421).

Definition 30. Let $F: \bar{A} \rightarrow \mathbb{R}, \nu \geq 0, n:=[\nu]$ such that $F(\cdot \omega) \in$ $A C^{n}\left(\left[R_{1}, R_{2}\right]\right)$, for all $\omega \in S^{N-1}$.

We call the left Caputo radial fractional derivative the following function

$$
\frac{\partial_{* R_{1}}^{\nu} F(x)}{\partial r^{\nu}}:=\frac{1}{\Gamma(n-\nu)} \int_{R_{1}}^{r}(r-t)^{n-\nu-1} \frac{\partial^{n} F(t \omega)}{\partial r^{n}} d t,
$$

where $x \in \bar{A}$, i.e. $x=r \omega, r \in\left[R_{1}, R_{2}\right], \omega \in S^{N-1}$.

Clearly

$$
\frac{\partial_{* R_{1}}^{0} F(x)}{\partial r^{0}}=F(x)
$$




$$
\frac{\partial_{* R_{1}}^{\nu} F(x)}{\partial r^{\nu}}=\frac{\partial^{\nu} F(x)}{\partial r^{\nu}}, \text { if } \nu \in \mathbb{N}
$$

Above function (119) exists almost everywhere for $x \in \bar{A}$, (see [1], p. 422). We mention the following fundamental representation result (see [1], p. 422-423 and [5]).

Theorem 31. Let $\nu \geq \gamma+1, \gamma \geq 0, n:=[\nu], m:=[\gamma], F: \bar{A} \rightarrow \mathbb{R}$ with $F \in L_{1}(A)$. Assume that $F(\cdot \omega) \in A C^{n}\left(\left[R_{1}, R_{2}\right]\right)$, for all $\omega \in S^{N-1}$, and that $\frac{\partial_{* R_{1}}^{\nu} F(\cdot \omega)}{\partial r^{\nu}} \in L_{\infty}\left(R_{1}, R_{2}\right)$ for all $\omega \in S^{N-1}$.

Further assume that $\frac{\partial_{* R_{1}}^{\nu} F(x)}{\partial r^{\nu}} \in L_{\infty}(A)$. More precisely, for these $r \in$ $\left[R_{1}, R_{2}\right]$, for each $\omega \in S^{N-1}$, for which $D_{* R_{1}}^{\nu} F(r \omega)$ takes real values, there exists $M_{1}>0$ such that $\left|D_{* R_{1}}^{\nu} F(r \omega)\right| \leq M_{1}$.

We suppose that $\frac{\partial^{j} F\left(R_{1} \omega\right)}{\partial r^{j}}=0, j=m, m+1, \ldots, n-1 ;$ for every $\omega \in$ $S^{N-1}$. Then

$$
\frac{\partial_{* R_{1}}^{\gamma} F(x)}{\partial r^{\gamma}}=D_{* R_{1}}^{\gamma} F(r \omega)=\frac{1}{\Gamma(\nu-\gamma)} \int_{R_{1}}^{r}(r-t)^{\nu-\gamma-1}\left(D_{* R_{1}}^{\nu} F\right)(t \omega) d t,
$$

valid $\forall x \in \bar{A}$; i.e. true $\forall r \in\left[R_{1}, R_{2}\right]$ and $\forall \omega \in S^{N-1} ; \gamma>0$.

Here

$$
D_{* R_{1}}^{\gamma} F(\cdot \omega) \in A C\left(\left[R_{1}, R_{2}\right]\right),
$$

$\forall \omega \in S^{N-1} ; \gamma>0$.

Furthermore

In particular, it holds

$$
\frac{\partial_{* R_{1}}^{\gamma} F(x)}{\partial r^{\gamma}} \in L_{\infty}(A), \gamma>0
$$

$$
F(x)=F(r \omega)=\frac{1}{\Gamma(\nu)} \int_{R_{1}}^{r}(r-t)^{\nu-1}\left(D_{* R_{1}}^{\nu} F\right)(t \omega) d t,
$$

true $\forall x \in \bar{A} ;$ i.e. true $\forall r \in\left[R_{1}, R_{2}\right]$ and $\forall \omega \in S^{N-1}$, and

$$
F(\cdot \omega) \in A C\left(\left[R_{1}, R_{2}\right]\right), \quad \forall \omega \in S^{N-1} .
$$

We finish with the following Poincaré type inequalities involving left Caputo radial fractional derivatives.

Theorem 32. Here all as in Theorem $31, p \geq 1$. Then

1)

$$
\left\|\frac{\partial_{* R_{1}}^{\gamma} F}{\partial r^{\gamma}}\right\|_{p, A} \leq\left(\frac{R_{2}}{R_{1}}\right)^{\left(\frac{N-1}{p}\right)}\left(\frac{\left(R_{2}-R_{1}\right)^{(\nu-\gamma)}}{\Gamma(\nu-\gamma+1)}\right)\left\|\frac{\partial_{* R_{1}}^{\nu} F}{\partial r^{\nu}}\right\|_{p, A},
$$


2) When $\gamma=0$, we have

$$
\|F\|_{p, A} \leq\left(\frac{R_{2}}{R_{1}}\right)^{\left(\frac{N-1}{p}\right)}\left(\frac{\left(R_{2}-R_{1}\right)^{\nu}}{\Gamma(\nu+1)}\right)\left\|\frac{\partial_{* R_{1}}^{\nu} F}{\partial r^{\nu}}\right\|_{p, A} .
$$

Proof. As in Theorem 25, based on Theorem 31.

See also similar results in $[1$, p. 464].

\section{REFERENCES}

[1] G. A. Anastassiou, Fractional Differentiation Inequalities, Research Monograph, Springer, New York, 2009.

[2] G. A. Anastassiou, On right fractional calculus, Chaos Solitons Fractals, 42 (2009), 365-376.

[3] G. A. Anastassiou, Balanced fractional Opial inequalities, Chaos Solitons Fractals, 42 (3) (2009), 1523-1528.

[4] G. A. Anastassiou, Fractional Korovkin theory, Chaos Solitons Fractals, 42 (2009), 2080-2094.

[5] G. A. Anastassiou, Fractional representation formulae and right fractional inequalities, Math. Comput. Modelling, 54 (11-12) (2011), 3098-3115.

[6] J. A. Canavati, The Riemann-Liouville Integral, Nieuw Arch. Wiskd., 5 (1) (1987), $53-75$.

[7] Kai Diethelm, The Analysis of Fractional Differential Equations, Lecture Notes in Mathematics, Vol 2004, 1st edition, Springer, New York, Heidelberg, 2010.

[8] A. M. A. El-Sayed and M. Gaber, On the finite Caputo and finite Riesz derivatives, Electron. J. Theor. Phys., 3 (12) (2006), 81-95.

[9] R. Gorenflo and F. Mainardi, Essentials of Fractional Calculus, 2000, Maphysto Center, http://www.maphysto.dk/oldpages/ events/LevyCAC2000/MainardiNotes/ fm2k0a.ps.

[10] G. D. Handley, J.J. Koliha and J. Pečarić, Hilbert-Pachpatte type integral inequalities for fractional derivatives, Frac. Calc. Appl. Anal, 4 (1) 2001, 37-46.

[11] H. G. Hardy, Notes on some points in the integral calculus, Messenger of Mathematics, 47 (10) 1918, 145-150.

[12] S. Iqbal, K. Krulic and J. Pecaric, On an inequality of H.G. Hardy, J. Inequal. Appl., Volume 2010, Article ID 264347, 23 pages.

[13] A. A. Kilbas, H. M. Srivastava and J. J. Trujillo, Theory and Applications of Fractional Differential Equations, vol. 204 of North-Holland Mathematics Studies, Elsevier, New York, NY, USA, 2006.

[14] T. Mamatov, S. Samko, Mixed fractional integration operators in mixed weighted Hölder spaces, Frac. Calc. Appl. Anal., 13, (3) (2010), 245-259.

[15] W. Rudin, Real and Complex Analysis, International Student Edition, Mc Graw Hill, London, New York, 1970.

[16] S. G. Samko, A. A. Kilbas and O. I. Marichev, Fractional Integral and Derivatives: Theory and Applications, Gordon and Breach Science Publishers, Yverdon, Switzerland, 1993. 
[17] D. Stroock, A Concise Introduction to the Theory of Integration, Third Edition, Birkhäuser, Boston, Basel, Berlin, 1999.

(Received: April 5, 2012)

Department of Mathematical Sciences University of Memphis

Memphis, TN 38152, U.S.A.

E-mail: ganastss@memphis.edu 